\title{
Homogenization-based stiffness optimization and projection of 2D coated structures with orthotropic infill
}

\author{
Groen, Jeroen P.; Wu, Jun; Sigmund, Ole
}

Published in:

Computer Methods in Applied Mechanics and Engineering

Link to article, DOI:

10.1016/j.cma.2019.02.031

Publication date:

2019

Document Version

Peer reviewed version

Link back to DTU Orbit

Citation (APA):

Groen, J. P., Wu, J., \& Sigmund, O. (2019). Homogenization-based stiffness optimization and projection of 2D coated structures with orthotropic infill. Computer Methods in Applied Mechanics and Engineering, 349, 722-742. https://doi.org/10.1016/j.cma.2019.02.031

\section{General rights}

Copyright and moral rights for the publications made accessible in the public portal are retained by the authors and/or other copyright owners and it is a condition of accessing publications that users recognise and abide by the legal requirements associated with these rights.

- Users may download and print one copy of any publication from the public portal for the purpose of private study or research.

- You may not further distribute the material or use it for any profit-making activity or commercial gain

- You may freely distribute the URL identifying the publication in the public portal 


\title{
Homogenization-based stiffness optimization and projection of 2D coated structures with orthotropic infill
}

\author{
Jeroen P. Groen ${ }^{\mathrm{a}, 1, *}$, Jun $\mathrm{Wu}^{\mathrm{b}}$, Ole Sigmund ${ }^{\mathrm{a}}$ \\ ${ }^{a}$ Department of Mechanical Engineering, Solid Mechanics, Technical University of Denmark \\ ${ }^{b}$ Department of Design Engineering, Delft University of Technology
}

\begin{abstract}
This paper concerns compliance minimization and projection of coated structures with orthotropic infill material in 2D. The purpose of the work is two-fold. First, we introduce an efficient homogenization-based approach to perform topology optimization of coated structures with orthotropic infill material. The design space is relaxed to allow for a composite material description, which means that designs with complex microstructures can be obtained on relatively coarse meshes. Second, a method is presented to project the homogenization-based designs on a fine but realizable scale. A novel method to adaptively refine the lattice structure is presented to allow for a regular spacing of the infill. Numerical experiments show excellent behavior of the projected designs, with structural performance almost identical to the homogenizationbased designs. Furthermore, a reduction in computational cost of at least an order of magnitude is achieved, compared to a related approach in which the infill is optimized using a density-based approach.
\end{abstract}

Keywords: Topology optimization, Coated structures, Homogenization, High-resolution, Infill

\section{Introduction}

Topology optimization is recognized as an important design method, with numerous applications in industry and academia. Furthermore, the flexibility offered by additive manufacturing (AM) methods makes topology optimization the ideal design method for this rapidly growing field. In recent years a large number of works have considered incorporation of constraints posed by the AM process directly into the optimization framework. Examples are geometric constraints to restrict the overhang angle (Langelaar, 2017; Qian, 2017; Gaynor and Guest, 2016; Allaire et al., 2017), and methods to restrict the length-scale of the design as well as to make them robust against manufacturing variations, see (Lazarov et al., 2016) for a detailed review of such methods. For a global overview on the state of the art and future trends in topology optimization for additive manufacturing, the reader is referred to (Liu et al., 2018).

Most additive manufacturing processes, such as Fused Deposition Modeling (FDM) work with a solid shell to represent the surface, reinforced by porous infill. The reason these so-called coated structures are considered instead of solid structures are high strength-to-weight ratio, good energy absorption characteristics, and high thermal and acoustic insulation properties (Gibson and Ashby, 1999). Furthermore, porous structures can alleviate thermal hot-spots that are prone to cause large stresses in printed designs (Allaire and Jakabčin, 2018; Ranjan et al., 2017). Compared to their solid counterparts, coated structures with porous infill can obtain significantly increased buckling stability (Clausen et al., 2016), as well as a better performance w.r.t. unpredicted loading conditions and material deficiency (Wu et al., 2018), at the cost of slightly increased compliance. We do; however, remind the reader that truss or lattice-like infill is inferior to close-walled cell structures when only considering stiffness, as e.g. discussed in (Sigmund et al., 2016).

\footnotetext{
* Correspondence to: J. P. Groen, Department of Mechanical Engineering, Solid Mechanics, Technical University of Denmark, Nils Koppels Allé, Building 404, 2800 Kgs. Lyngby, Denmark

${ }^{1}$ E-mail: jergro@mek.dtu.dk
} 
On the other hand, here we consider the 2D-case where the differentiation between open- and closed-walled structures does not directly apply.

Traditionally, the porous infill in coated structures consists of repetitive infill patterns (e.g., triangles and hexagons). However, Wu et al. (2018) proposed a density-based method to design bone-inspired microstructures as porous infill. In another approach Clausen et al. $(2015,2017)$ presented a method to optimize a coated structure, using a solid shell and an isotropic base material that can be interpreted as a uniform porous infill. There, density-based optimization was applied and using successive filtering operations a clear distinction between coating and infill material could be made. In a natural subsequent step these methods have been combined to concurrently design both the coating and the infill (Wu et al., 2017). Similar to density-based methods, level-set methods have been used to design for material interface properties (Vermaak et al., 2014; Wang and Kang, 2018). This approach was recently extended to first design a coating layer, and subsequently an infill (Dapogny et al., 2019). Besides the computational mechanics community, the computer graphics community has recently proposed many works on the optimization of porous structures, e.g. (Lu et al., 2014; Martínez et al., 2016; Wu et al., 2016).

In this work we extend the method of Clausen et al. (2015) to design coated structures with a macroscopically varying orthotropic infill material. The design space is relaxed to allow for a composite material description, which means that designs with complex microstructures can be obtained on relatively coarse meshes, e.g. $300 \times 100$ elements as is shown in Figure 1(a). To describe the periodic infill composite we make use of the well-known square unit-cell with a rectangular hole (Bendsøe and Kikuchi, 1988), shown in Figure 1(b). Recent studies (Groen and Sigmund, 2017; Allaire et al., 2018) inspired by Pantz and Trabelsi $(2008,2010)$ have shown that microstructures with a rectangular hole can be projected on a much finer mesh to obtain manufacturable designs, thereby avoiding the need for separation of scales. Our work is continuing on the projection procedure presented in (Groen and Sigmund, 2017; Larsen et al., 2018), where an explicit constraint is used to align the projected microstructure with the directions of lamination. To keep the unit-cell spacing as regular as possible we also introduce a novel scheme that adaptively refines the periodicity as can be seen in Figure 1(c), where a resolution of $3000 \times 1000$ elements is used.

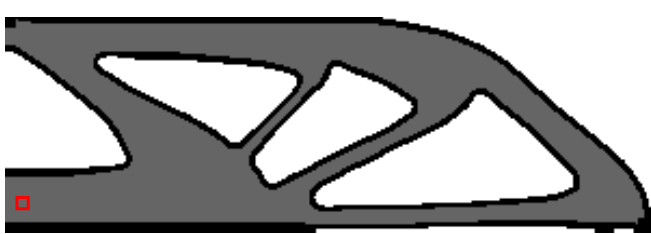

(a) Homogenization-based topology optimization resolution: $300 \times 100$ elements.

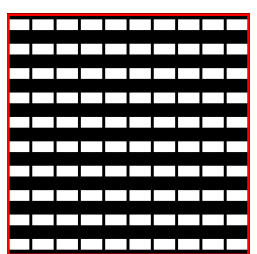

(b) Microstructure

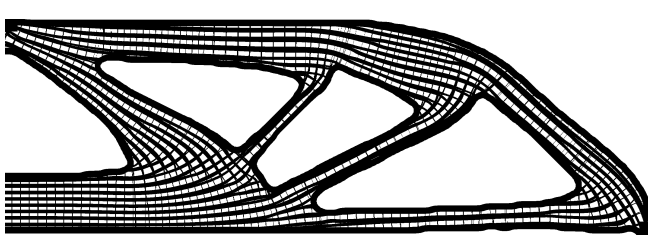

(c) Projection of composite design on a fine mesh resolution: $3000 \times 1000$ elements.

Figure 1: Overview of the proposed methodology to obtain high-resolution coated designs, with composite orthotropic infill.

The paper is organized in two parts. The first part encompasses homogenization-based topology optimization for coated structures. The theory is introduced in Section 2, while numerical experiments regarding performance of the optimized results and comparisons to the use of isotropic infill material are discussed in Section 3. The second part of this article deals with the projection method to obtain high-resolution manufacturable structures on a fine mesh. The theory and implementation will be discussed in Section 4, while numerical experiments on the performance and numerical efficiency of the projected structures are discussed in Section 5. Finally, Section 6 contains the most important conclusions of this study.

\section{Homogenization-based topology optimization for coated structures}

A coated structure consists of two material phases, a coating and an infill as can be seen in Figure 1(a). Clausen et al. (2015) introduced an elegant method to obtain a coated structure using only a single field as design variable. Successive filter and projection operations allowed for a clear distinction between coating, 
isotropic infill and void. The use of composite infill material, introduced in this work, will add extra design variables. Nevertheless, the method to distinguish between infill and coating remains almost the same.

\subsection{Successive filter operations}

The procedure to distinguish between coating and infill makes use of two well-established filter methods in topology optimization. The first is a smoothing operation using the density filter. The second is a projection step to force the smoothed values on the interval $[0,1]$ towards either 0 or 1 .

\subsubsection{Smoothing}

As basis for subsequent projection we use the Helmholtz-type PDE-based density filter (Lazarov and Sigmund, 2011),

$$
-\left(\frac{R}{2 \sqrt{3}}\right)^{2} \nabla^{2} \tilde{\phi}+\tilde{\phi}=\phi .
$$

Here scalar $R$ corresponds to the length-scale imposed by the filter operation. $\phi$ is the unfiltered field, while $\tilde{\phi}$ is the filtered field. Homogeneous Neumann boundary conditions are applied at the boundary of the filter domain. A discussion on undesired boundary effects of standard filter methods will be given later in this section.

\subsubsection{Projection}

Projection methods have been successfully applied in topology optimization to obtain black-and-white designs (Guest et al., 2004; Sigmund, 2007). Here we use the formulation for the smoothed Heaviside projection proposed by Wang et al. (2011),

$$
\overline{\tilde{\phi}}=\frac{\tanh (\beta \eta)+\tanh (\beta(\tilde{\phi}-\eta))}{\tanh (\beta \eta)+\tanh (\beta(1-\eta))} .
$$

Here $\overline{\tilde{\phi}}$ is the projected field. $\beta$ determines the steepness of the projection, i.e. when $\beta \rightarrow \infty$ a sharp step is modeled. In general, a continuation approach is used for $\beta$, i.e. a low value is used during the first iterations, after which $\beta$ is gradually increased. Furthermore, $\eta \in[0,1]$ is the threshold parameter where $\eta>0.5$ corresponds to an erosion operation, while $\eta<0.5$ corresponds to a dilation operation.

\subsubsection{Combining the filters to obtain a coated structure}

Analogous to Clausen et al. (2015) we use successive filter operations to obtain field $\varphi$ describing the base structure and field $\tau$ describing the coating. Additional variables, which will be introduced in Section 2.2, are used to describe the shape of the infill. However, these variables do not affect the distinction between coating $\Omega_{c}$, infill $\Omega_{l}$ or void $\Omega_{v}$ regions.

$$
\mathbf{x} \in\left\{\begin{array}{llll}
\Omega_{v} & \text { if } & \varphi(\mathbf{x})=0 & \text { and if } \tau(\mathbf{x})=0 \\
\Omega_{l} & \text { if } & \varphi(\mathbf{x})=1 & \text { and if } \tau(\mathbf{x})=0 \\
\Omega_{c} & \text { if } & \tau(\mathbf{x})=1 . &
\end{array}\right.
$$

The successive smoothing projection and gradient operations used in this work can be seen in Figure 2 .

The design field is filtered and projected twice using filter radius $R_{1}$, and projection parameters $\beta_{1}$ and $\eta_{1}$, which indirectly control the length-scale of $\varphi$. The base structure which is now either 0 or 1 , is smoothed again using $R_{2}<R_{1}$, such that the resulting field $\tilde{\varphi}$ has smooth boundaries. The coating layer can then be defined by taking the Euclidean norm of the spatial gradients of $\tilde{\varphi} \cdot\|\nabla \tilde{\varphi}\|$ is then normalized such that the largest possible gradient norm corresponds to 1 . For this we use a normalization factor $\alpha$, which is related to $R_{2}$ using (Clausen et al., 2015),

$$
\alpha=\frac{R_{2}}{\sqrt{3}} .
$$




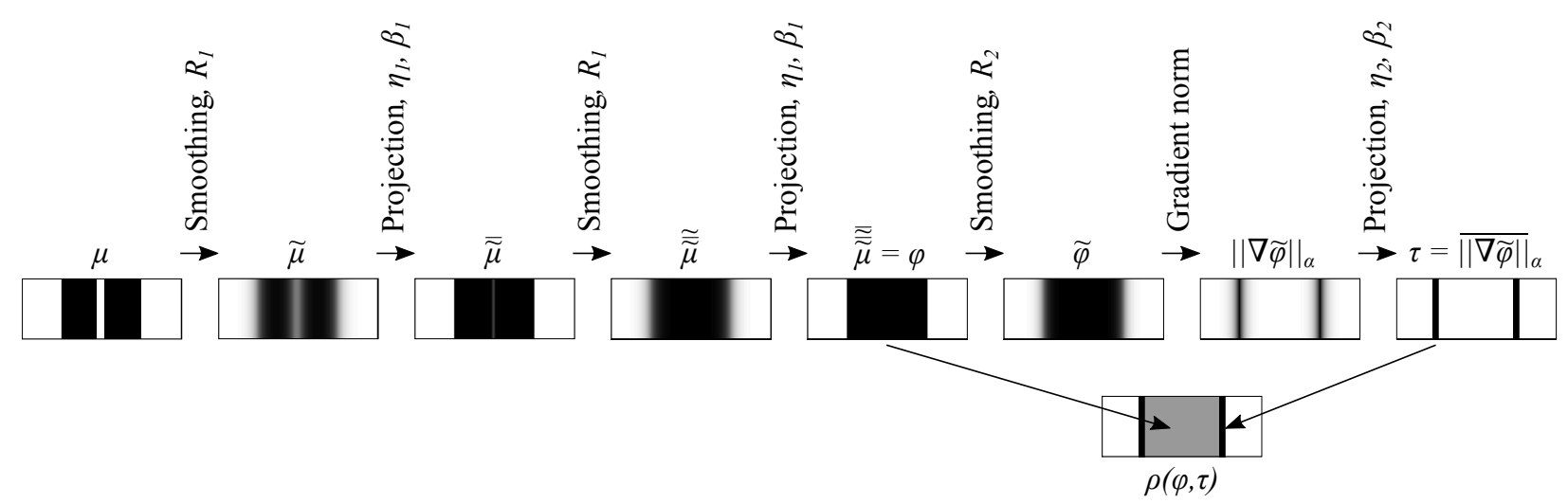

Figure 2: Subsequent filtering steps, allowing to separate the base structure $\varphi$ and the $\operatorname{coating} \tau$.

The choice of notation for this normalized gradient norm $\|\nabla \tilde{\varphi}\|_{\alpha}$ might seem odd; however, it is used to be consistent with the work of Clausen et al. (2015) and Wu et al. (2017). Hence, the term shall be read as the Euclidean norm of $\nabla \tilde{\varphi}$ divided by scalar $\alpha$, rather than as the usual definition, which would be the $L^{\alpha}{ }_{-}$ norm of $\nabla \tilde{\varphi}$.

The normalized gradient norm $\|\nabla \tilde{\varphi}\|_{\alpha}$ is subsequently projected using $\beta_{2}$ and $\eta_{2}$ to define the coating

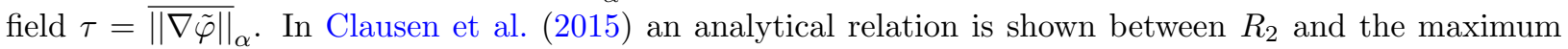
coating thickness. This is used to select $R_{2}$ for a user-specified coating thickness $t_{r e f}$ as,

$$
R_{2}=\frac{\sqrt{3}}{\ln (2)} t_{r e f} \approx 2.5 t_{r e f}
$$

The motivation for using a double smoothing and projection (DSP) approach for the design field is that numerical experiments using a single smoothing and projection (SSP) approach showed the possibility that fields $\varphi$ and $\tau$ describing the base structure and coating, did not converge exactly to 0 or 1 . A similar observation can be made from Figure 13 in Clausen et al. (2015), where it is difficult to distinguish between infill and void. To circumvent this undesired effect we use the DSP approach as proposed by Christiansen et al. (2015). More details on the effect of this DSP approach versus the SSP approach used in Clausen et al. (2015) will be given in Section 3.

\subsubsection{Note on the filter boundary conditions}

It is well-known that the use of homogeneous Neumann boundary conditions used in the smoothing operation of Equation 1 causes artifacts on the optimized design near the domain boundary. To circumvent this undesired effect, we make use of the domain extension approach proposed by Clausen and Andreassen (2017). In this approach the physical domain is padded using void elements $(\mu=0)$, except at boundaries at which the displacement field is constrained. The extension distance $d_{e x t}>R_{1}$ is chosen large enough, such that the homogeneous Neumann filter boundary conditions do not affect the final design. All filtering operations, finite element analysis and objective and constraint calculations are performed on this extended domain. An overview of the design domain $\Omega$ and boundary conditions for the MBB-beam example including the extended domain can be seen in Figure 3.

Solid blocks of material are used at the two boundary conditions. This guarantees a minimum feature size of twice the coating thickness, e.g. $2 t_{r e f}$, and reinforces the structure thus preventing load concentrations. Furthermore, the load is applied in a distributed fashion over the complete top of the solid block, while the displacement constraint is applied in an average sense over the bottom of the lower block. This is done to prevent load concentrations when the optimized structure is mapped on a finer mesh, as will be discussed later on.

The domain extension approach works well for robust topology optimization problems or three-field SIMP optimization problems (density filtering, followed by projection), using $\eta=0.5$. However, in the 


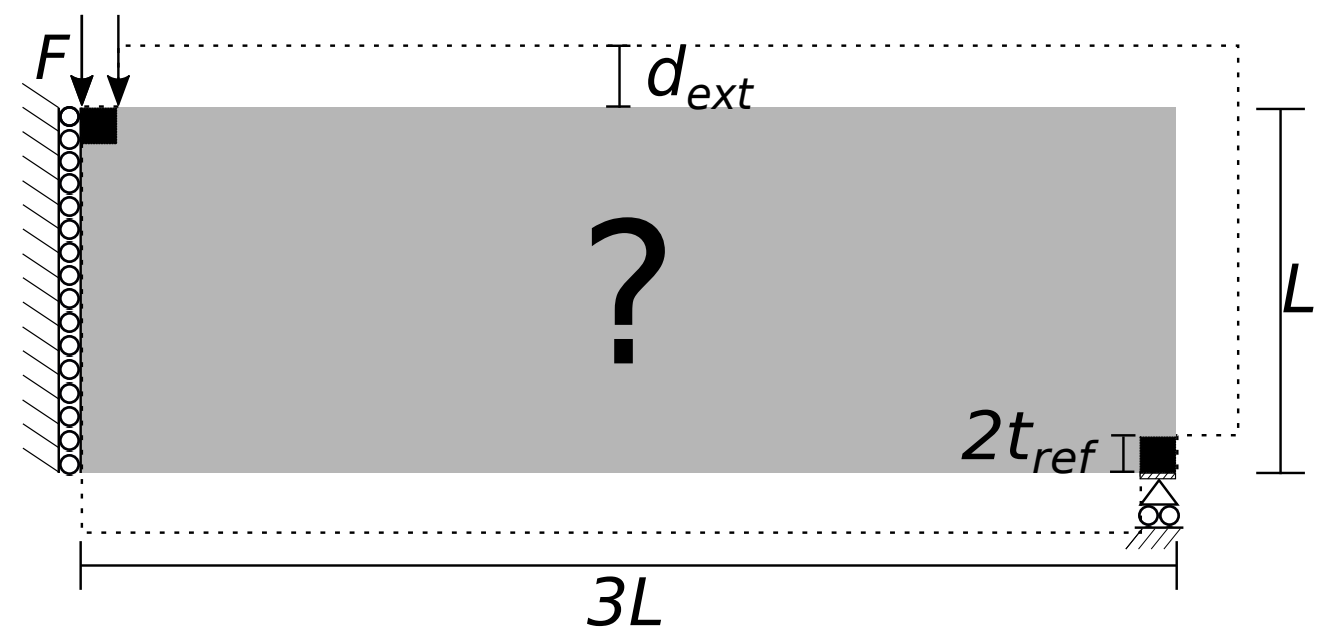

Figure 3: Design domain $\Omega$ and boundary conditions of the MBB-beam example. $\Omega$ is indicated using gray material, while the extended filter domain is bounded by the dotted line.

context of coated structures, it is possible that half of the coating $\left(t_{r e f} / 2\right)$ can exceed the design domain. To make sure that the optimized structure is within the bounds of $\Omega$, the elasticity tensor in the padded domain is multiplied by penalization parameter $q<1$, while $q=1 \in \Omega$. Numerical experiments have shown that the use of $q=0.2$ effectively restricts optimized structures to $\Omega$.

\subsection{Interpolation of elastic properties and density}

For the numerical examples we restrict ourselves to coating and infill made from the same material, with Young's modulus $E^{0}=1$, Poisson's ratio $\nu^{0}=0.3$, and mass density $m^{0}=1$; however, extension to different coating and infill material is trivial. As infill we chose to use the square unit-cell with rectangular hole introduced by Bendsøe and Kikuchi (1988), shown in Figure 4.
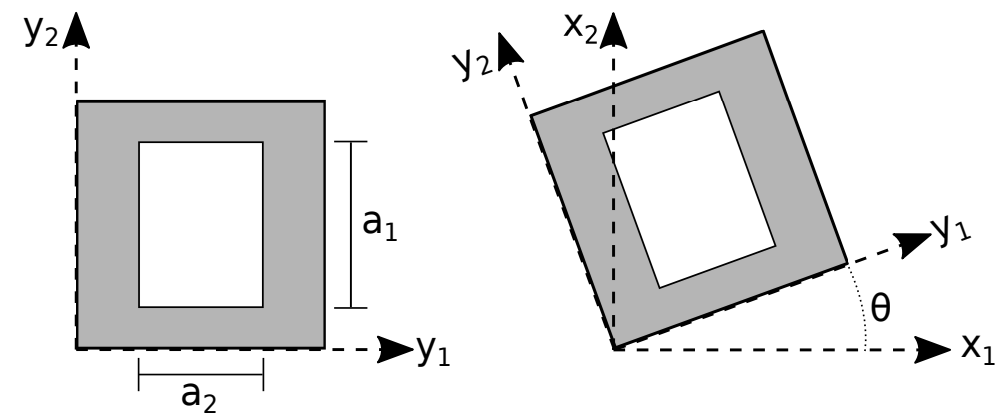

Figure 4: Layout of the unit-cell with a rectangular hole, in local $\left(\mathrm{y}_{1}, \mathrm{y}_{2}\right)$, and global $\left(\mathrm{x}_{1}, \mathrm{x}_{2}\right)$ coordinate system.

The constitutive properties which are close in performance to the optimal rank-2 laminate are obtained using numerical homogenization. A database of the constitutive properties in the local frame for a large number of different combinations for the height $a_{1}$ and width $a_{2}$ of the hole has been created. Afterwards, we can interpolate the effective properties $\mathbf{E}^{H}$ and sensitivities for any combination of $a_{1}$ and $a_{2}$. The corresponding elasticity tensor of the infill in the global frame of reference $\mathbf{E}^{I}$ can be calculated as,

$$
\mathbf{E}^{I}\left(\theta, a_{1}, a_{2}\right)=\mathbf{R}(\theta) \mathbf{E}^{H}\left(a_{1}, a_{2}\right) \mathbf{R}(\theta)^{T},
$$

with $\mathbf{R}$ being the well-known frame rotation matrix using angle $\theta$ describing the angle between material and global frame of reference. The mass fraction of the microstructure $m^{I}$ is given as,

$$
m^{I}=1-a_{1} a_{2} .
$$


With the properties of the coating and infill known, we can define the interpolation of the density $\rho$ and elasticity tensor $\mathbf{E}$ throughout the design domain. To this end, we use material indicator $\varphi$ and coating indicator $\tau$, such that,

$$
\rho\left(\varphi, \tau, a_{1}, a_{2}\right)=m^{I}\left(a_{1}, a_{2}\right) \varphi+\left(1-m^{I}\left(a_{1}, a_{2}\right) \varphi\right) \tau .
$$

Similarly, the local elasticity tensor $\mathbf{E}$ can be defined.

$$
\mathbf{E}\left(\varphi, \tau, \theta, a_{1}, a_{2}\right)=10^{-9} \mathbf{E}^{0}+q\left(\mathbf{E}^{I}\left(\theta, a_{1}, a_{2}\right)-10^{-9} \mathbf{E}^{0}\right) \varphi^{p_{1}}+q\left(\mathbf{E}^{0}-\mathbf{E}^{I}\left(\theta, a_{1}, a_{2}\right) \varphi^{p_{1}}\right) \tau^{p_{2}},
$$

where, $\mathbf{E}^{0}$ is the elasticity tensor for the isotropic coating material, and $p_{1}$ and $p_{2}$ are penalty parameters to penalize intermediate values of $\varphi$ and $\tau$. Please note that the model using isotropic infill from Clausen et al. (2015) can easily be recovered from Equations 8 and 9.

\subsection{Problem formulation}

We focus on the minimization of a compliance functional $\mathcal{J}$ for plane stress, single load-case problems under the assumption of linear elasticity. We discretize the design domain by bi-linear finite elements, and the material properties are assumed to be element-wise constant. The microstructures of the infill are described by design vectors $\mathbf{a}_{1}, \mathbf{a}_{2}, \boldsymbol{\theta}$ which determine the width and height of the hole as well as the unit-cell orientation, while the coating and base structure are defined by $\boldsymbol{\mu}$.

The topology optimization problem is solved in nested form. For each design iteration the equilibrium equations are satisfied by FE-analysis. As shown by Pedersen (1989, 1990), the optimal orientation of an orthotropic composite coincides with the principal stress directions, hence the unit-cell orientation $\boldsymbol{\theta}$ is updated in each minimization step after the displacement and corresponding stress calculation. Subsequently, design vectors $\boldsymbol{\mu}, \mathbf{a}_{1}$ and $\mathbf{a}_{2}$ are updated based on their gradients using the updated microstructure orientation. The discretized optimization problem can thus be written as,

$$
\begin{aligned}
\min _{\boldsymbol{\mu}, \mathbf{a}_{1}, \mathbf{a}_{2}, \boldsymbol{\theta}} & : \mathcal{J}\left(\boldsymbol{\mu}, \mathbf{a}_{1}, \mathbf{a}_{2}, \boldsymbol{\theta}, \mathbf{U}\right), \\
\text { s.t. } & : \mathbf{K}\left(\boldsymbol{\mu}, \mathbf{a}_{1}, \mathbf{a}_{2}, \boldsymbol{\theta}\right) \mathbf{U}=\mathbf{F}, \\
& : \mathbf{v}^{T} \boldsymbol{\rho}\left(\boldsymbol{\mu}, \mathbf{a}_{1}, \mathbf{a}_{2}\right)-V_{\max } A \leq 0, \\
& : \mathbf{a}_{l} \leq \mathbf{a}_{1}, \mathbf{a}_{2} \leq \mathbf{a}_{u}, \\
& : \mathbf{0} \leq \boldsymbol{\mu} \leq \mathbf{1},
\end{aligned}
$$

where $\mathbf{v}$ is the vector containing the element volumes, and $V_{\text {max }}$ is the maximum allowed volume fraction of the material in the design domain, where $A$ is the area of $\Omega$. Stiffness matrix $\mathbf{K}$ is a function of $\boldsymbol{\mu}$, $\mathbf{a}_{1}, \mathbf{a}_{2}$, and $\boldsymbol{\theta} . \mathbf{F}$ describes the loads acting on the domain, and $\mathbf{U}$ describes the solution of the equilibrium equation. The vectors $\mathbf{a}_{l}$ and $\mathbf{a}_{u}$ on interval $[0,1]$ describe the lower and upper bound on the hole size of the microstructure, subject to $\mathbf{a}_{u}>\mathbf{a}_{l}$. For the design update of $\boldsymbol{\mu}, \mathbf{a}_{1}$ and $\mathbf{a}_{2}$ the MATLAB implementation of the Method of Moving Asymptotes (MMA) introduced by Svanberg (1987) is used.

The formulation above is the most general formulation. We allow full freedom for the microstructures, within bounds $\mathbf{a}_{l}$ and $\mathbf{a}_{u}$. To avoid fully void or solid infill material we use $\mathbf{a}_{l} \geq 0.1$ and $\mathbf{a}_{u} \leq 0.9$, where the exact values are problem dependent. In some optimization examples we do not want to exploit the full design freedom of the microstructures. Therefore, we present in total 4 different problem formulations that will be used throughout this work; however, note that more problem formulations can be envisioned.

\section{Problem 0: Fixed microstructure density, isotropic microstructure}

This is the original coating approach as proposed in Clausen et al. (2015). We specify an infill mass fraction $m^{I}$, and choose a stiffness for the isotropic microstructure $E^{I}$ that satisfies the upper bound of the Hashin-Shtrikman bounds (Hashin and Shtrikman, 1963),

$$
E^{I}=\frac{m^{I}}{3-2 m^{I}} .
$$


Problem 1: Fixed microstructure density, equal widths of hole

This is the simplest problem using the orthotropic microstructure. The infill has a user-defined mass fraction $m^{I}$, and both widths are set equal such that,

$$
a_{i}=\sqrt{1-m^{I}}, \quad i=1,2 .
$$

Problem 2: Fixed microstructure density, variable widths of hole

Again we have a user-defined mass fraction $m^{I}$. However, $a_{1}$ and $a_{2}$ are allowed to vary such that $m^{I}$ is always satisfied. Hence, we need one design variable to describe the shape of the rectangular hole. For this we use variable $a_{1}$, such that

$$
a_{2}=\frac{1-m^{I}}{a_{1}}
$$

Furthermore, we can set box-constraints $\mathbf{a}_{l}$ and $\mathbf{a}_{u}$ on $\mathbf{a}_{1}$ to restrict the set of allowed microstructures. Here we always use an upper bound of $\mathbf{a}_{u}=0.9$, after which we calculate $\mathbf{a}_{l}$ which will be larger than 0.1 .

Problem 3: Variable microstructure density, variable widths of hole

This is the most general optimization problem, which is the problem shown in Equation 10, with variables $\boldsymbol{\mu}, \mathbf{a}_{1}$ and $\mathbf{a}_{2}$ updated using the MMA. This means that contrary to the previous problems, the microstructure density can vary. To avoid artificially stiff checkerboard-like patterns of infill material, we here need to regularize design vectors $\mathbf{a}_{1}$ and $\mathbf{a}_{2}$ into $\tilde{\mathbf{a}}_{1}$ and $\tilde{\mathbf{a}}_{2}$ describing the physical size of the hole using a density filter with a filter radius just larger than the finite element size.

\subsection{Sensitivity analysis}

The sensitivity of the self-adjoint objective function $\mathcal{J}$ with respect to any design variable $x$ can be derived as,

$$
\frac{\partial \mathcal{J}}{\partial x}=-\mathbf{U}^{T} \frac{\partial \mathbf{K}}{\partial x} \mathbf{U}=-\mathbf{U}^{T} \frac{\partial \mathbf{K}}{\partial x} \mathbf{U}=-\sum_{j} \mathbf{u}_{j}^{T} \int_{\Omega_{j}} \mathbf{B}^{T} \frac{\partial \mathbf{E}_{j}}{\partial x} \mathbf{B} \mathrm{d} \Omega_{j} \mathbf{u}_{j},
$$

where $j$ indicates the set of elements for which the elasticity tensor $\mathbf{E}_{j}$ is influenced by $x$ due to filter operations.

\subsubsection{Sensitivities w.r.t. $\mu$}

The sensitivity of compliance $\mathcal{J}$ w.r.t the variable determining the base structure of element $e\left(\varphi_{e}\right)$ is,

$$
\frac{\partial \mathcal{J}}{\partial \varphi_{e}}=-\mathbf{u}_{e}^{T} \int_{\Omega_{e}} \mathbf{B}^{T} \frac{\mathrm{d} \mathbf{E}_{e}}{\mathrm{~d} \varphi_{e}} \mathbf{B} \mathrm{d} \Omega_{e} \mathbf{u}_{e}-\sum_{k} \mathbf{u}_{k}^{T} \int_{\Omega_{k}} \mathbf{B}^{T} \frac{\mathrm{d} \mathbf{E}_{k}}{\mathrm{~d} \tau} \frac{d \tau}{d \varphi_{e}} \mathbf{B} \mathrm{d} \Omega_{k} \mathbf{u}_{k}
$$

Here $k$ indicates the set of elements for which the elasticity tensor $\mathbf{E}_{k}$ is influenced by $\varphi_{e}$ due to filter operations. Furthermore,

$$
\begin{gathered}
\frac{\mathrm{d} \mathbf{E}_{e}}{\mathrm{~d} \varphi_{e}}=q p_{1}\left(\mathbf{E}^{I}\left(\theta, a_{1}, a_{2}\right)-10^{-9} \mathbf{E}^{c}\right) \varphi_{e}^{p_{1}-1}-q p_{1} \mathbf{E}^{I}\left(\theta, a_{1}, a_{2}\right) \varphi_{e}^{p_{1}-1} \tau^{p_{2}} \\
\frac{\mathrm{d} \mathbf{E}_{k}}{\mathrm{~d} \tau}=q p_{2}\left(\mathbf{E}^{c}-\mathbf{E}^{I}\left(\theta, a_{1}, a_{2}\right) \varphi^{p_{1}}\right) \tau^{p_{2}-1}
\end{gathered}
$$

The derivative of coating field $\tau$ w.r.t the element base structure $\varphi_{e}$ is omitted here, but can be found in Clausen et al. (2015). With the derivatives of the objective w.r.t $\varphi$ known, it is easy to get the derivatives w.r.t design field $\mu$. These are standard chain rule modifications for the two smoothing and two projection steps. These expressions are well-known and can be found in Lazarov and Sigmund (2011) and Wang et al. (2011). 


\subsubsection{Sensitivities w.r.t. $a_{i}$}

For an optimization problem of type 3 (full freedom), where both $a_{1}$ and $a_{2}$ are design variables, we can write the sensitivity of the elasticity tensor w.r.t the filtered variable determining the size of the hole of element $e$ as,

$$
\frac{\partial \mathbf{E}_{e}}{\partial \tilde{a}_{e, 1}}=q \mathbf{R}_{e} \frac{\partial \mathbf{E}_{e}^{H}}{\partial \tilde{a}_{e, 1}} \mathbf{R}_{e}^{T} \varphi_{e}^{p_{1}}-q \mathbf{R}_{e} \frac{\partial \mathbf{E}_{e}^{H}}{\partial \tilde{a}_{e, 1}} \mathbf{R}_{e}^{T} \varphi_{e}^{p_{1}} \tau^{p_{2}} .
$$

The derivative of the homogenized elasticity tensor w.r.t. the parameter describing the height of the hole $\tilde{a}_{e, 1}$ is obtained by taking the derivative of the interpolation basis, used for the interpolation of the homogenized elasticity tensor. The standard chain rule modification to get the derivative w.r.t the design variable $a_{1}$ is again trivial.

For an optimization problem of type 2, where we only have one variable per element to describe the shape of the hole $a_{1}$ we can write,

$$
\frac{\partial \mathbf{E}_{e}}{\partial a_{e, 1}}=q \mathbf{R}_{e}\left(\frac{\partial \mathbf{E}_{e}^{H}}{\partial a_{e, 1}}-\frac{1-m^{I}}{a_{e, 1}^{2}} \frac{\partial \mathbf{E}_{e}^{H}}{\partial a_{e, 2}}\right) \mathbf{R}_{e}^{T} \varphi_{e}^{p_{1}}-q \mathbf{R}_{e}\left(\frac{\partial \mathbf{E}_{e}^{H}}{\partial a_{e, 1}}-\frac{1-m^{I}}{a_{e, 1}^{2}} \frac{\partial \mathbf{E}_{e}^{H}}{\partial a_{e, 2}}\right) \mathbf{R}_{e}^{T} \varphi_{e}^{p_{1}} \tau^{p_{2}} .
$$

\subsection{Parameters to control the optimization problem}

An overview of all parameters that are kept the same in all numerical examples in this work can be seen in Table 1.

Table 1: The parameters that are used in all numerical examples in this work.

\begin{tabular}{ccc}
\hline$p_{1}$ & 3 & Stiffness penalization of base structure $\varphi$ \\
$p_{2}$ & 1 & Stiffness penalization of coating indicator $\tau$ \\
$\eta_{1}$ & 0.5 & Threshold parameter used to obtain $\varphi$ \\
$\eta_{2}$ & 0.5 & Threshold parameter used to obtain $\tau$ \\
$\beta_{\text {start }}$ & 2 & Starting steepness parameter for the projection \\
$\beta_{\text {end }}$ & 128 & Final steepness parameter for the projection \\
$q$ & 0.2 & Penalization of elasticity tensor in padded domain \\
\hline
\end{tabular}

These parameter choices have been extensively motivated using numerical experiments. For example, the choice for not penalizing the coating $\left(p_{2}=1\right)$ might seem counter-intuitive at first sight; however, numerical examples using $p_{2}>1$ yielded solutions that more easily ended up in worse local minima. For the steepness parameter of the projection operation we use $\beta_{1}=\beta_{2}$, which we start at $\beta_{\text {start }}$ and double every $30-100$ iterations until $\beta_{\text {end }}$. The exact number if iterations before $\beta$ is doubled depends on whether the maximum change in each design variable becomes less than 0.01 .

\section{Numerical examples for topology optimization of coated structures}

For the numerical experiments in this paper we focus on the MBB-beam example shown in Figure 3. Furthermore, we use the bridge example for which the loads and boundary conditions, including padded domain are shown in Figure 5.

\subsection{Experiments on the MBB-beam example}

For the experiments on the MBB-beam example we use a discretization of $300 \times 100$ finite elements. A volume constraint of $V_{\max }=0.4, R_{1}=0.15 \mathrm{~L}$ and $t_{r e f}=0.03 \mathrm{~L}$ is used. The problem formulations with the fixed microstructure density (problems 0-2), have been used to optimize the structure for various infill volume fractions $\left(m^{I}=0.4, m^{I}=0.5, m^{I}=0.6, m^{I}=0.7, m^{I}=0.8\right.$ and $\left.m^{I}=0.9\right)$. The corresponding compliance values on the coarse optimization mesh $\mathcal{J}^{c}$ can be seen in Table 2.

It can be seen that the compliances $\mathcal{J}^{c}$ become lower as more freedom is introduced in the microstructures, i.e. going from problem 0 to 2 . The corresponding density distributions for optimized results using $m^{I}=0.5$ are shown in Figure 6. 


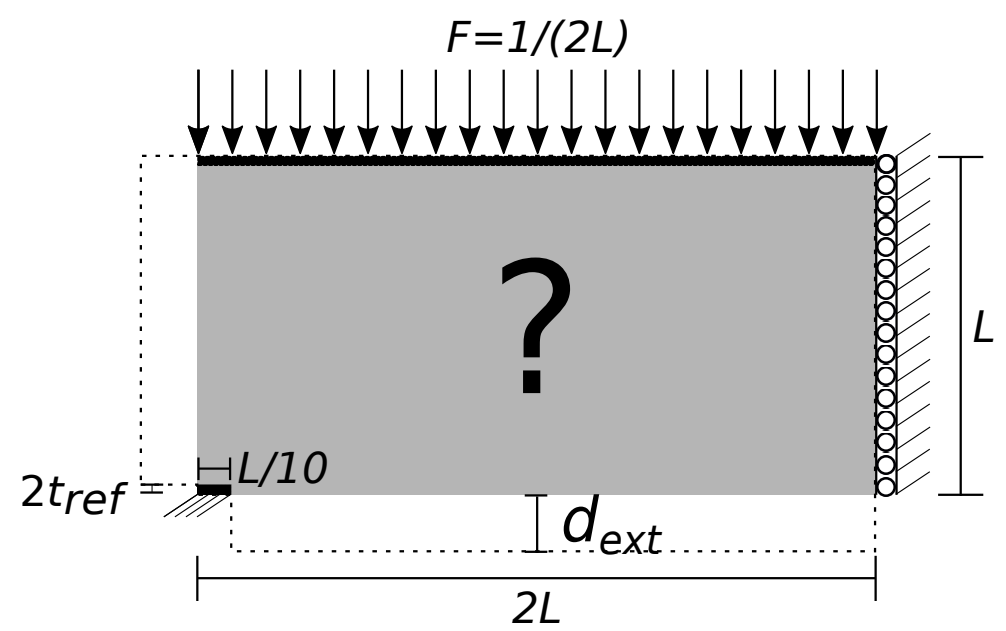

Figure 5: Design domain $\Omega$ and boundary conditions of the bridge example. $\Omega$ is indicated using gray material, while the extended filter domain is bounded by the dotted line.

Table 2: Compliance $\mathcal{J}^{c}$ for different problem formulations and different infill densities $m^{I}$. Label DSP indicates a double smoothing and projection approach is used to obtain $\varphi$, while label SSP indicates that a single smoothing and projection step is used.

\begin{tabular}{lcccccccc}
\hline & & $m^{I}=0.4$ & $m^{I}=0.5$ & $m^{I}=0.6$ & $m^{I}=0.7$ & $m^{I}=0.8$ & $m^{I}=0.9$ & $m^{I}=1.0$ \\
\hline Problem form 0 & DSP & 362.83 & 325.94 & 297.78 & 279.80 & 256.68 & 237.63 & 215.53 \\
Problem form 1 & DSP & 318.45 & 291.14 & 274.92 & 266.85 & 251.38 & 236.37 & 215.53 \\
Problem form 2 & DSP & 267.92 & 247.52 & 234.30 & 227.02 & 219.67 & 217.73 & 215.53 \\
\hline Problem form 0 & SSP & 360.45 & 325.75 & 291.49 & 273.20 & 254.35 & 236.55 & 213.57 \\
Problem form 1 & SSP & 315.42 & 292.41 & 274.16 & 258.53 & 245.42 & 235.16 & 213.57 \\
Problem form 2 & SSP & 266.02 & 246.83 & 231.44 & 224.44 & 220.19 & 217.84 & 213.57 \\
\hline
\end{tabular}

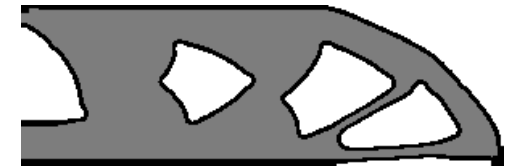

(a) Problem form 0 .

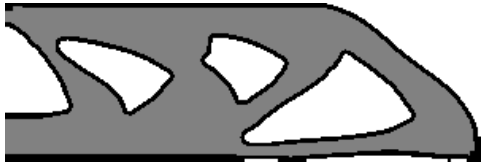

(b) Problem form 1

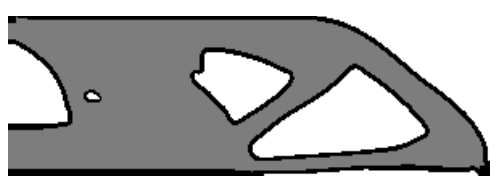

(c) Problem form 2.

Figure 6: Density distributions for the MBB-beam example using $m^{I}=0.5$ and optimized for different problem formulations using the double smoothing and projection (DSP) approach.

From Table 2 it is clear that for larger infill densities the structures converge to the results of a threefield SIMP approach (SIMP with smoothing and projection). This can also be seen in Figure 7(a) and (b). The MBB-beam optimized using three-field SIMP with these filter settings has a corresponding compliance $\mathcal{J}^{c}=213.57$, and can be seen in Figure 7(c).

The structures using a single smoothing and projection (SSP) approach seem to have a slightly better compliance. However, this is at the cost of undetermined coating and infill, as seen in Figures 8(a) and (b), where small features of void can be observed, that are not bounded by coating. The reason for vanishing coating can be seen in Figures 8(c) and (d). At locations where the coating is vanishing the field describing the base structure $\varphi$ has non-binary values. Since there is no sharp transition from 0 to 1 , the corresponding normalized gradient norm $\|\nabla \tilde{\varphi}\|_{\alpha}$ is smaller than threshold $\eta_{2}$, resulting in a void region without coating. The DSP approach greatly reduces the possibility of $\varphi$ being non-binary.

We cannot guarantee that a double smoothing and projection (DSP) approach will result in binary 


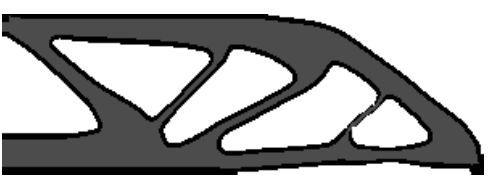

(a) Problem form 1, DSP, $m^{I}=0.7$.

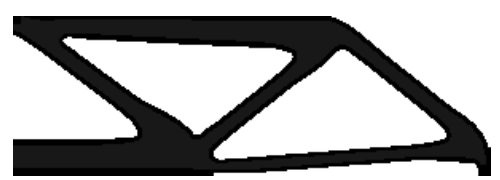

(b) Problem form 1, DSP, $m^{I}=0.9$.

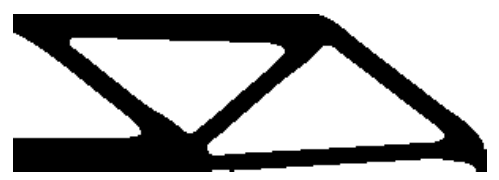

(c) Three-field SIMP (SSP) $m^{I}=1$.

Figure 7: Density distributions for the MBB-beam example optimized for different infill volume fractions $m^{I}$.

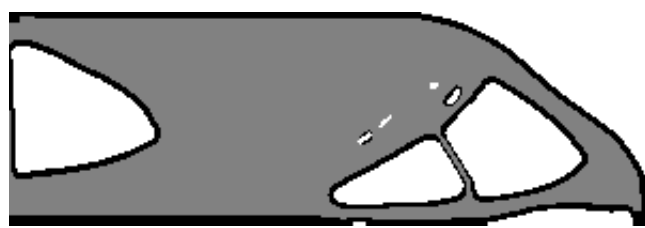

(a) Problem form $1, m^{I}=0.5$.

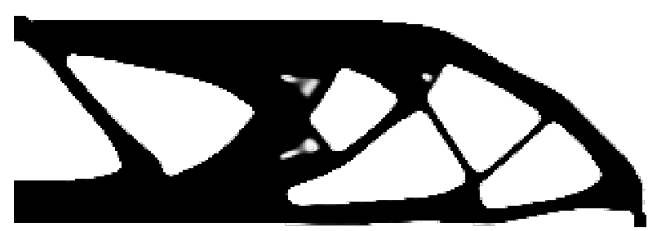

(c) $\varphi$ corresponding to $m^{I}=0.6$.

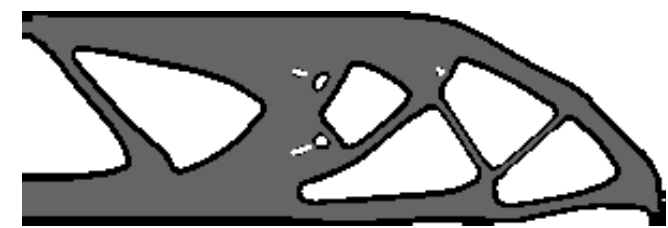

(b) Problem form $1, m^{I}=0.6$.

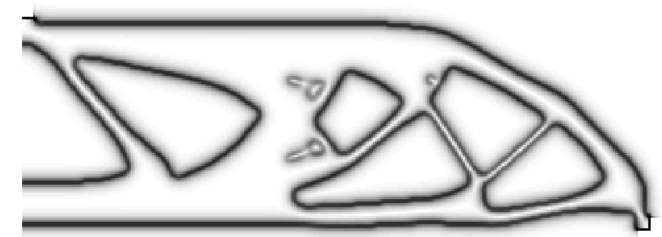

(d) $\|\nabla \tilde{\varphi}\|_{\alpha}$ corresponding to $m^{I}=0.6$.

Figure 8: Density distributions for the MBB-beam example optimized using a single smoothing and projection (SSP) step.

features in base structure and coating fields $\varphi$ and $\tau$. However, numerical experiments have shown a reduction of around $90 \%$ in the occurrence of vanishing coating compared to the SSP approach. The rare cases where a void region is not bounded by coating is when there is a thin feature of $\varphi=0$. These thin features result in $\tilde{\varphi}$ not going to zero in the middle of this void feature, and consequently the corresponding normalized gradient norm $\|\nabla \tilde{\varphi}\|_{\alpha}$ is smaller than threshold $\eta_{2}$. A large number of numerical experiments have been performed to test the effects of threshold parameters $\eta_{1}, \eta_{2}$ and filter radii $R_{1}$ and $R_{2}$. Unfortunately, changing thresholds $\eta_{1}$ and $\eta_{2}$ only results in reduced performance.

To show the effect of changing coating thickness $t_{r e f}$ and filter radius $R_{1}$, the density distribution using problem form 1, a DSP approach and $m^{I}=0.5$ can be seen in Figures 9(a) and (b) for a thicker coating $t_{r e f}=0.06 L$, and 2 values of $R_{1}$. As can be seen, increasing $t_{r e f}$ results in more coating compared to the amount of infill material, which defies the goal of the present work (to design coated structures). In general, the coating thickness is uniform; however, in case of a small feature it can be seen that the coating thickness reduces. Especially in combination with a low value of $R_{1}$, there is an increased possibility of a vanishing coating. Hence, it is recommended to have $R_{1}>R_{2}$.

A possible remedy to avoid the problem of vanishing coatings completely can be to apply image processing after homogenization-based optimization and explicitly enforce coating if required. An alternative approach could be to strongly enforce the length-scale of the coating using the method of Zhou et al. (2015). Testing both these ideas will be the topic of future work.

Finally, when we use the optimization problem with full freedom (problem 3), an even better performing structure than using standard three-field SIMP can be obtained. In Table 3 one can find the compliance values corresponding to several lower $a_{l}$ and upper $a_{u}$ bounds on the parameters that describe the shape of the hole.

It can be seen that the lowest compliance can be reached when there is the largest freedom for parameters $a_{i}$ to vary. To illustrate this, consider the density distribution shown in Figure 10 (a). Here the microstruc- 


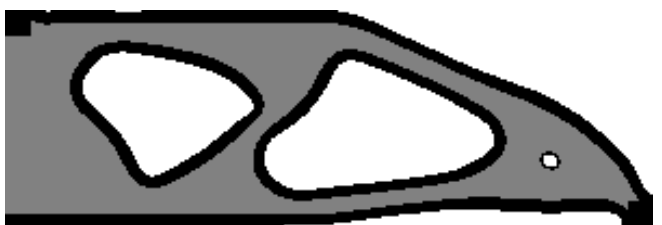

(a) $R_{1}=0.30 L, R_{2}=0.15 L, \mathcal{J}^{c}=262.44$.

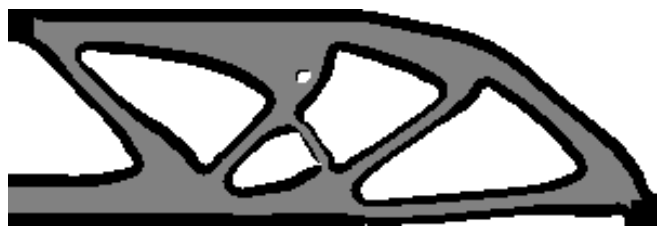

(b) $R_{1}=0.15 L, R_{2}=0.15 L, \mathcal{J}^{c}=241.60$

Figure 9: Density distributions for the MBB-beam example optimized using problem form 1, a DSP approach and $m^{I}=0.5$ for a coating thickness of $t_{r e f}=0.06 L$, and different filter radii $R_{1}$.

Table 3: Compliance $\mathcal{J}^{c}$ for problem formulations 3 using the DSP and SSP approach for various bounds on the shape of the microstructure.

\begin{tabular}{ccccccccccc}
\hline$a_{l}$ & 0.2 & 0.2 & 0.3 & 0.3 & 0.4 & 0.4 & 0.5 & 0.5 & 0.6 & 0.6 \\
$a_{u}$ & 0.8 & 0.9 & 0.8 & 0.9 & 0.8 & 0.9 & 0.8 & 0.9 & 0.8 & 0.9 \\
\hline DSP & 222.81 & 207.12 & 228.70 & 212.42 & 236.29 & 219.35 & 245.61 & 229.48 & 259.57 & 245.15 \\
SSP & 226.91 & 207.09 & 231.91 & 212.32 & 238.04 & 219.38 & 248.50 & 229.34 & 261.96 & 244.97 \\
\hline
\end{tabular}

tures are allowed to be nearly solid $a_{l}=0.2$ and also get close to void $a_{u}=0.9$. Hence, there is no need to create holes. However, if the bounds are set a bit tighter, e.g. $a_{l}=0.3$ and $a_{u}=0.8$ holes will originate in the optimized structure, as can be seen in Figure 10 (b).

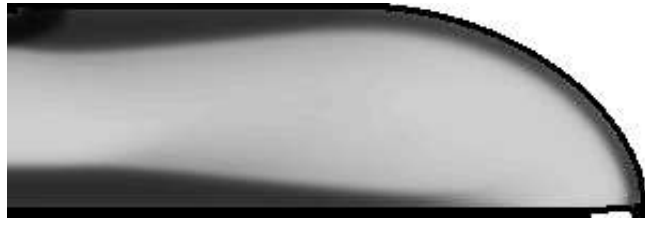

(a) Problem form 3, DSP, $a_{l}=0.2$ and $a_{u}=0.9$.

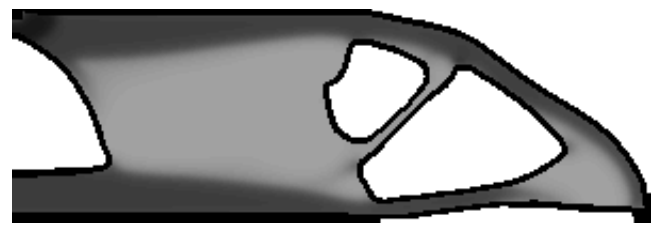

(b) Problem form 3, DSP, $a_{l}=0.3$ and $a_{u}=0.8$.

Figure 10: Density distribution for the MBB-beam example optimized with DSP approach for the most general optimization problem, shown for various bounds on the shape of the microstructure.

\subsection{Bridge design example}

The second optimization example considered is the bridge design example, shown in Figure 5. The optimization is performed on a coarse mesh of $200 \times 100$ elements. A volume constraint of $V_{\max }=0.2$, $R_{1}=0.15 L$ and $t_{r e f}=0.03 L$ are used. The optimized designs and their compliance values, for $m^{I}=0.5$, and for problem forms 0-2 are shown in Figure 11 (a)-(c). The design optimized for problem form 3, using $a_{l}=0.2$ and $a_{u}=0.8$ is shown in Figure 11 (d). Finally, it is noted that this example also has been solved using a three-field SIMP approach, the corresponding compliance of such a design is 24.50.

It is interesting to note that the two small void areas in the top of Figure 11 (d) are not bounded by coating material. This is a rare case where even the DSP approach cannot guarantee coating everywhere. Although this does not happen in many cases, it is still an undesired effect.

\section{Projecting coated structures with microstructures as infill}

To project the infill as a smooth and continuous lattice structure, two mapping functions $\phi_{1}$ and $\phi_{2}$ have to be determined, representing the two orthogonal layers of the sequence of unit-cells (Pantz and Trabelsi, 2008, 2010; Groen and Sigmund, 2017). These mapping functions are then later used to project the composite shape of the microstructures on a fine mesh. We will not go into full detail on the derivation of 


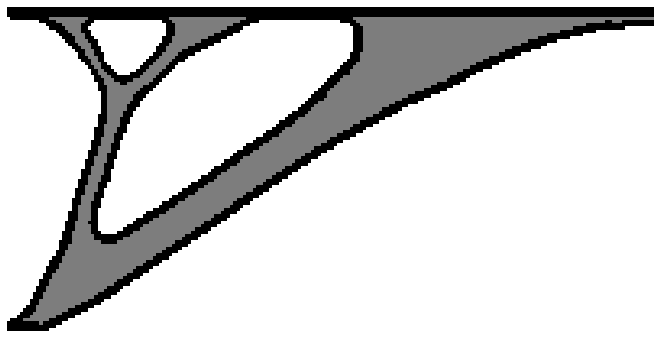

(a) Problem form $0, \mathcal{J}^{c}=34.08$.

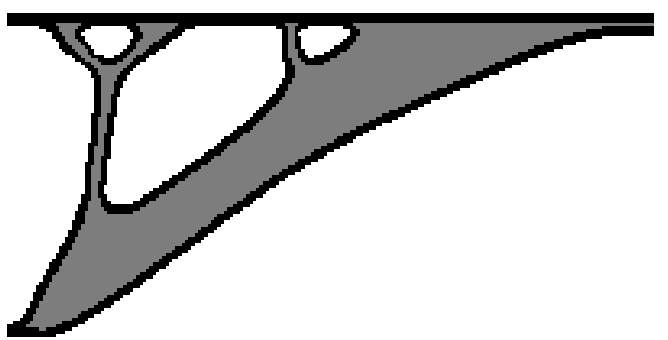

(c) Problem form 2, $\mathcal{J}^{c}=27.26$.

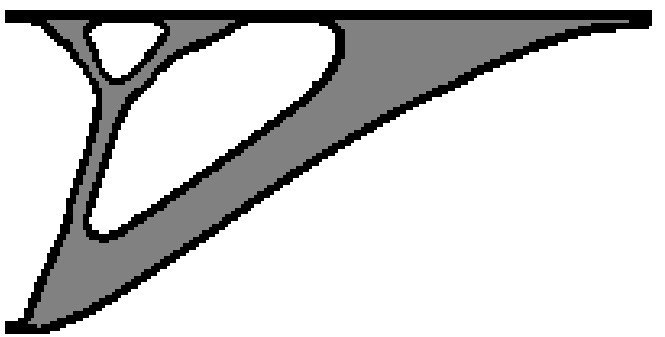

(b) Problem form 1, $\mathcal{J}^{c}=30.63$.

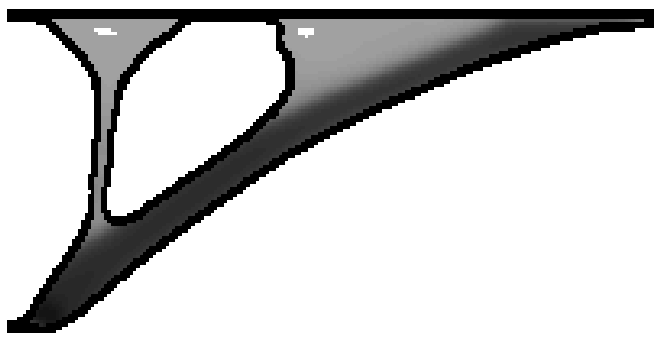

(d) Problem form 3, $\mathcal{J}^{c}=26.13$.

Figure 11: Density distribution for the bridge design example, for all 4 types of optimization problems.

the mapping functions; instead we focus on changes and improvements compared to the approach presented in Groen and Sigmund (2017).

\subsection{Projecting a periodic composite shape}

Contrary to previous approaches we do not solve for the mapping function in the void part of $\Omega$, i.e. we create a conformal, albeit regular mesh on $\Omega_{m}=\Omega_{c} \bigcup \Omega_{l}$. Both mapping functions $\phi_{1}$ and $\phi_{2}$ can be obtained independently of each other; therefore, we restrict ourselves to the derivation of $\phi_{1}$. A suitable parameterization of $\phi_{1}$ has to fulfill:

1. $\phi_{1}$ should be constant in the direction perpendicular to the layer normal $\mathbf{n}_{1}$.

2. The spacing between the contour lines of $\phi_{1}$, should be as regular as possible without violating the first requirement.

To solve for $\phi_{1}$ we use the following minimization problem,

$$
\begin{aligned}
\min _{\phi_{1}(\mathbf{x})}: & \mathcal{I}\left(\phi_{1}(\mathbf{x})\right)=\frac{1}{2} \int_{\Omega_{m}}\left\|\nabla \phi_{1}(\mathbf{x})-\mathbf{n}_{1}(\mathbf{x})\right\|^{2} \mathrm{~d} \Omega_{m}, \\
\text { s.t. }: & \nabla \phi_{1}(\mathbf{x}) \cdot \mathbf{t}_{1}(\mathbf{x})=0 .
\end{aligned}
$$

Here $\mathbf{t}_{1}$ is tangential to normal vector $\mathbf{n}_{1}$, hence both depend on the local directions of lamination $\theta$,

$$
\mathbf{n}_{1}(\mathbf{x})=\mathbf{t}_{2}(\mathbf{x})=\left[\begin{array}{c}
-\sin (\theta(\mathbf{x})) \\
\cos (\theta(\mathbf{x}))
\end{array}\right], \quad \mathbf{n}_{2}(\mathbf{x})=\mathbf{t}_{1}(\mathbf{x})=\left[\begin{array}{c}
\cos (\theta(\mathbf{x})) \\
\sin (\theta(\mathbf{x}))
\end{array}\right] .
$$

It has to be noted that the parameter describing the direction of lamination $\theta$ is rotationally symmetric, hence there may be jumps of size $\pi$ in the angle field $\theta$. These jumps are identified using connected component labeling and aligned consistently as suggested in Groen and Sigmund (2017), to allow for a smooth projection using Equation 20.

The mapping functions can then be used to project the optimized shape. As opposed to Groen and Sigmund (2017) that used a cosine we here use a triangle wave function $\mathcal{S}$ using the sawtooth function in MATLAB.

$$
\tilde{\rho_{1}}(\mathbf{x})=\frac{1}{2}+\frac{1}{2} \mathcal{S}\left(P_{1} \phi_{1}(\mathbf{x})\right),
$$


where $P_{1}$ is a periodicity scaling parameter. The exact widths of the microstructure are then projected using Heaviside function $H$,

$$
\rho_{1}(\mathbf{x})=H\left(\tilde{\rho}_{1}(\mathbf{x})-\left(1-a_{1}(\mathbf{x})\right)\right) .
$$

After solving for the densities of both layers independently, they can be combined to obtain the density field $\rho$,

$$
\rho(\mathbf{x})=\max \left(\rho_{1}(\mathbf{x})+\rho_{2}(\mathbf{x})+\tau, 1\right) .
$$

The mapping problem is solved using bi-linear finite elements on an intermediate mesh using element size $h^{i}=h^{c} / 2$, on which angle field $\theta$ (after consistent alignment) is interpolated using linear interpolation. The constraint is not enforced explicitly; but in penalty form using penalty parameter $\gamma$. For large values of $\gamma$ the constraint enforces mapping functions $\phi_{1}$ and $\phi_{2}$ to be aligned with $\theta$, at the cost of relaxed periodicity.

Locally we can identify the spacing of the mapping by making use of the Euclidean norm of the derivatives of the mapping functions $\left\|\nabla \phi_{i}\right\|$. If the value of $\left\|\nabla \phi_{i}\right\|>1$, then the corresponding layer distance is locally compressed, similarly if $\left\|\nabla \phi_{i}\right\|<1$ the corresponding layer distance is locally stretched. In general we would like to impose an average layer distance $\varepsilon$. To do that we can determine periodicity scaling parameter $P_{i}$ as,

$$
P_{i}=\frac{2 \pi \int_{\Omega_{m}} \mathrm{~d} \Omega_{m}}{\varepsilon \int_{\Omega_{m}}\left\|\nabla \phi_{i}(\mathbf{x})\right\| \mathrm{d} \Omega_{m}} .
$$

After scaling, the mapping functions are interpolated on a fine mesh where $h^{f} \leq h^{c} / 10$, and the microstructure can be projected using Equations 22-24. The normalized gradient norm $\|\nabla \tilde{\varphi}\|_{\alpha}$ is interpolated using linear interpolation from coarse to fine mesh, afterwards the projection to obtain a clear coating $\tau=\overline{\| \nabla \tilde{\varphi}}_{\alpha}$ is performed.

To demonstrate the mapping procedure consider the simple test case shown in Figure 12(a). Here we have a coated structure on coarse mesh $\mathcal{T}^{c}$ consisting of $50 \times 50$ coarse elements, where the coating layer is exactly 1 element wide. The square microstructure has $a_{1}=a_{2}=0.9$ and the corresponding angle field is shown in Figure 12(b). The mapping is performed on $\mathcal{T}^{i}$ using $100 \times 100$ elements, where $\phi_{1}$ and $\phi_{2}$ are shown in Figure 12(c) and (d). The corresponding projection on $\mathcal{T}^{f}$ consisting of $1000 \times 1000$ elements is shown in Figure $12(\mathrm{e})$.

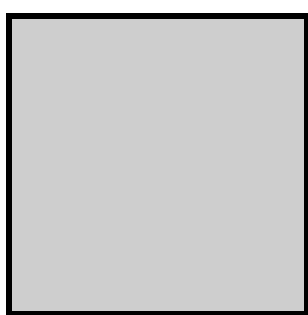

(a) $\rho$ on $\mathcal{T}^{c}$.

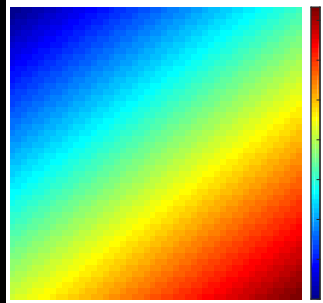

(b) $\theta$ on $\mathcal{T}^{c}$

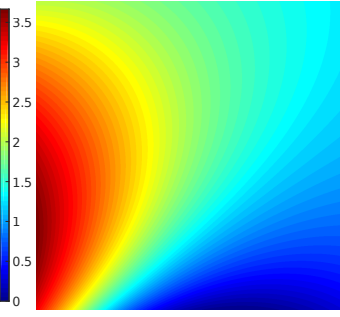

(c) $\phi_{1}$ on $\mathcal{T}^{i}$.

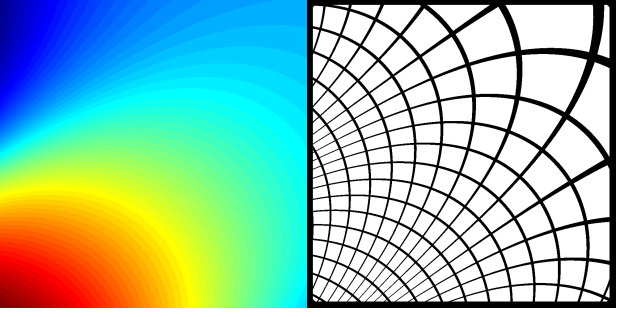

(d) $\phi_{2}$ on $\mathcal{T}^{i}$ (e) Projected $\rho$ on $\mathcal{T}^{f}$

Figure 12: Example of the mapping procedure, using $\varepsilon=80 h^{f}$. To demonstrate the use of a coarse $\mathcal{T}^{c}$, intermediate $\mathcal{T}^{i}$ and fine mesh $\mathcal{T}^{f}$.

\subsection{Method to adaptively refine the periodicity}

Figure 12(d) shows a large variation in the layer spacing throughout the domain. However, for performance, and manufacturability of 3D printed structures, a regular spacing is desired. A possible solution to obtain a more regular spacing is to relax the angle constraint by using a small value for $\gamma$; however, this results in a decreased performance. Close inspection of optimization results from Wu et al. (2017) (where the infill is freely optimized using SIMP) shows that structural members tend to split in two to counter increasing spacing between structural members. This observation leads to the idea of locally adapting the periodicity, to have a layer spacing as close to $\varepsilon$ as possible. 
Instead of using Equation 22 we use a function such that the periodicity is adapted using discrete periodicity scaling parameter $\lambda_{i}$, corresponding to the $i$-th mapping function, to obtain

$$
\left.\tilde{\rho}_{i}\left(\mathbf{x}, \lambda_{i}(\mathbf{x})\right)=\frac{1}{2}+\frac{1}{2} \mathcal{S}\left(2^{\left(\lambda_{i}(\mathbf{x})+1\right)} \pi \phi_{i}(\mathbf{x})+\lambda_{i}(\mathbf{x}) \pi\right)\right) .
$$

When $\lambda_{i}=0$, we have the same function as Equation 22 with $\varepsilon=1$. However, when $\lambda_{i}=1$, we have the same function as when $\varepsilon=0.5$. Hence, the periodicity is doubled. To get the periodicity in Equation 26 as close to $\varepsilon$ as possible we choose $\lambda_{i}$ as,

$$
\lambda_{i}(\mathbf{x})=\operatorname{round}\left(\log \left(\frac{1}{\varepsilon\left\|\nabla \phi_{i}(\mathbf{x})\right\|}\right) \frac{1}{\log (2)}\right) .
$$

A plot of $\lambda_{1}$ corresponding to $\phi_{1}$ in Figure 12(c) can be seen in Figure 13(a); similarly, $\lambda_{2}$ can be seen in Figure 13(b). The corresponding projection using Equation 26 can be seen in Figure 13(c), where it can be seen that the effective lattice spacing is now bounded to the interval $\left[\varepsilon 2^{-1 / 2}, \varepsilon 2^{1 / 2}\right]$.

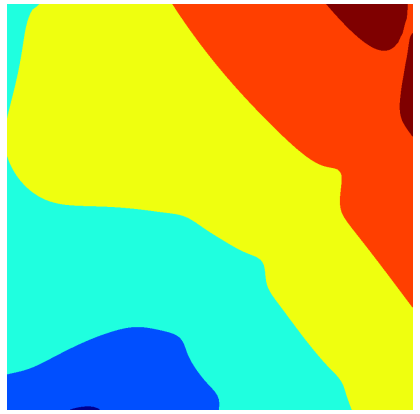

(a) $\lambda_{1}$ on $\mathcal{T}^{f}$.

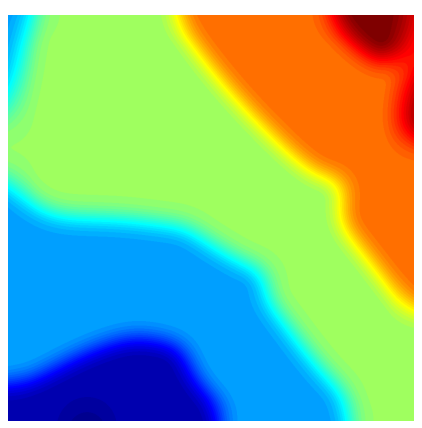

(d) $\tilde{\lambda}_{1}$ on $\mathcal{T}^{f}$.

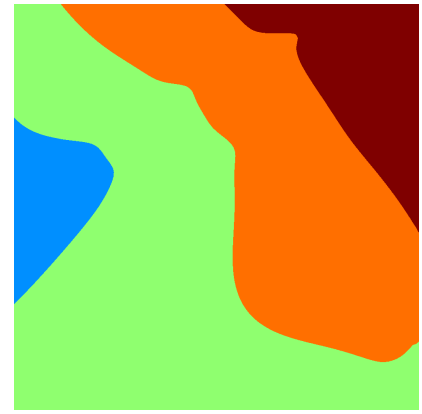

(b) $\lambda_{2}$ on $\mathcal{T}^{f}$

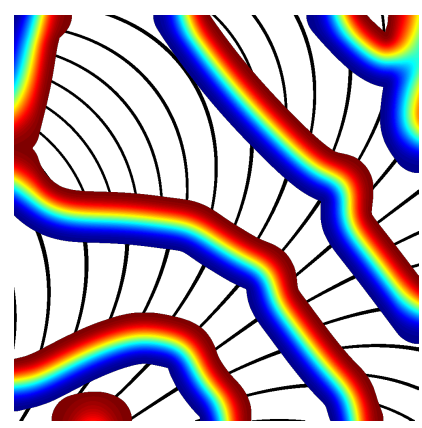

(e) Projected $\rho_{1}$ and $\psi_{1}$ on $\mathcal{T}^{f}$

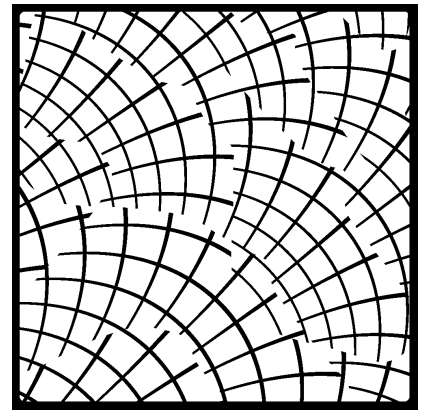

(c) Projected $\rho$ on $\mathcal{T}^{f}$.

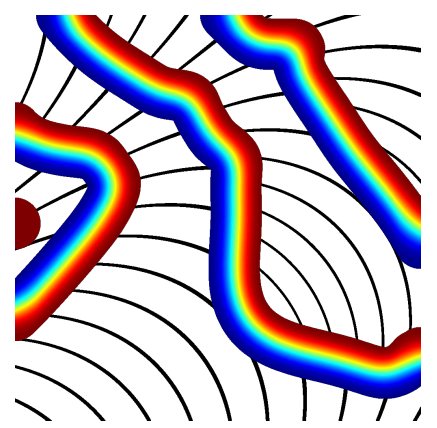

(f) Projected $\rho_{2}$ and $\psi_{2}$ on $\mathcal{T}^{f}$.

Figure 13: Visual explanation of adaptive periodicity and required transition zone, to split the structural members for $R^{*}=0.8 \varepsilon$.

Unfortunately however, the projected structure in Figure 13(c) is now discontinuous. To reconnect the bars a transition zone $\Omega_{T, i}$ is needed. To this end, we use a convolution operation to obtain $\tilde{\lambda}_{i}$, shown in Figure 13(d), using a linearly decaying convolution kernel with radius $R^{*}$. Using $\tilde{\lambda_{i}}$, we can determine splitting parameter $\psi_{i}$, which is used to identify if we are in the transition zone between two discrete values of $\lambda_{i}$.

$$
\psi_{i}(\mathbf{x})=\operatorname{modulo}\left(\tilde{\lambda}_{i}(\mathbf{x}), 1\right)
$$

If $\psi_{i}=0$, then we are outside $\Omega_{T, i}$ and we can use Equation 26, as shown in Figure 13(e) and (f), while inside $\Omega_{T, i}$ the value of $\psi_{i}$ is shown.

In $\Omega_{T, i}$ a structural member splits from a low periodicity $\left(\operatorname{floor}\left(\tilde{\lambda}_{i}\right)\right)$ to a higher periodicity $\left(\operatorname{ceil}\left(\tilde{\lambda_{i}}\right)\right)$, where both corresponding functions $\tilde{\rho}_{i}$ are shown in Figure 14(a). To model this splitting of members inside 
$\Omega_{T, i}$, we create function $\mathcal{F}_{i}$, which is a function of $\phi_{i}, \tilde{\lambda}_{i}$, and $\psi_{i} . \mathcal{F}_{i}$ consists of a base wave, on the interval $[0,2]$. This base wave is defined as $\tilde{\rho}_{i}$ for a low periodicity $\left(i . e\right.$. floor $\left.\left(\tilde{\lambda_{i}}\right)\right)$ multiplied by 2 , from which a part of width $1 / 2 \psi_{i}$ is subtracted as is shown for $\psi_{i}=0.5$ in Figure 14(b). Afterwards, another sawtooth-like wave is added to create $\mathcal{F}_{i}$. The distance between the peaks in $\mathcal{F}_{i}$ linearly increases with $\psi_{i}$, as can be seen in Figures 14(c)-(d).

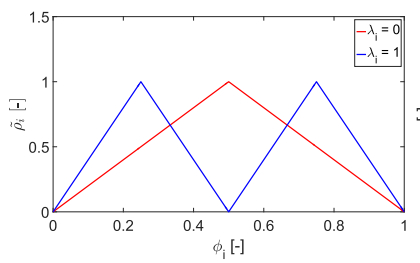

(a) $\tilde{\rho}_{i}$ for $\lambda_{i}=0$ and $\lambda_{i}=1$.

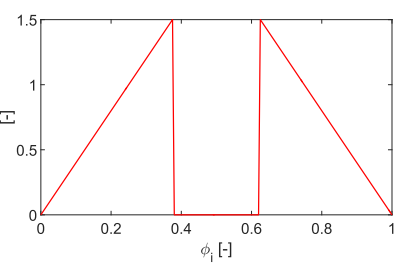

(b) Base minus $1 / 2 \psi_{i}$.

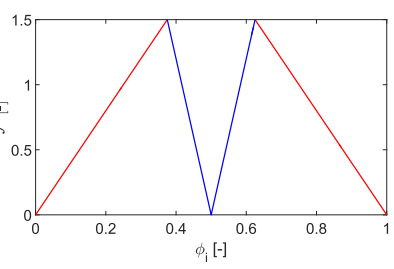

(c) $\mathcal{F}_{i}$ for $\psi_{i}=0.5$.

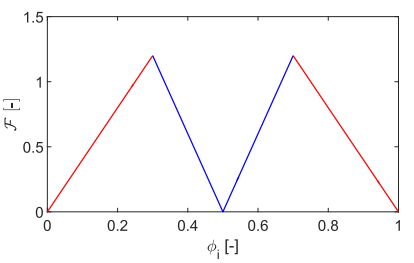

(d) $\mathcal{F}_{i}$ for $\psi_{i}=0.8$

Figure 14: Functions used in the transition zone to let a structural member split up into two members.

It has to be mentioned that Equation 23 cannot be used to impose the exact widths on $\mathcal{F}_{i}$ to obtain $\rho_{i}$. The reason is that, due to the choice of base wave, the peak of $\mathcal{F}_{i}$ is close to 2 for $\psi \rightarrow 0$. Hence, we need to numerically determine the threshold for the Heaviside function that we use to obtain $\rho_{i}$ from $\mathcal{F}_{i}$, for a given combination of $a_{i}$ and $\psi_{i}$. To do so, a bi-section scheme is used. The projection of the structure from Figure 12 using adaptive periodicity scaling can be seen for different values of $R^{*}$ in Figure $15(\mathrm{a})$-(c). Here it can be observed that increasing $R^{*}$ leads to a smoother transition for splitting of structural members. Numerical experiments have shown that the compliance of projected designs is the lowest when the transition goes as smooth as possible. Hence, $R^{*}=1.6 \varepsilon$ has been found to give the best results.

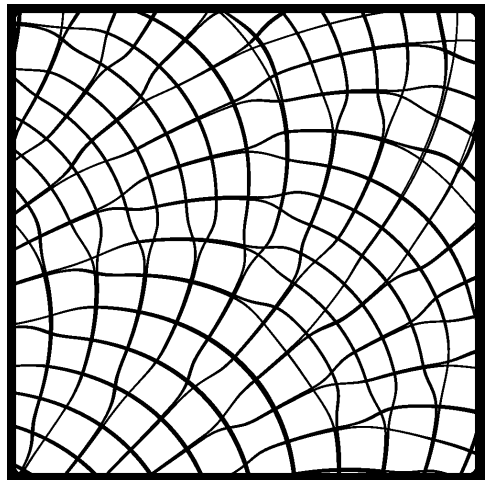

(a) $R^{*}=0.8 \varepsilon$

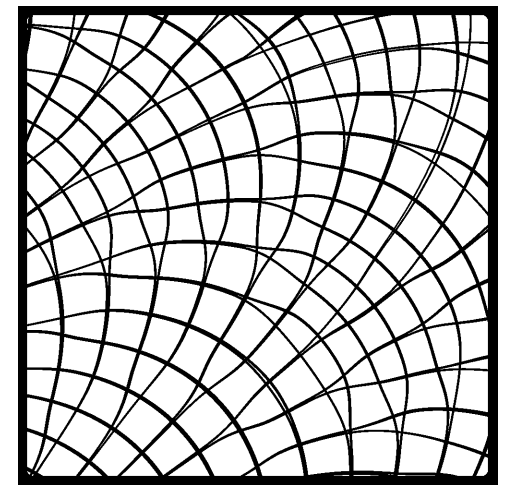

(b) $R^{*}=1.2 \varepsilon$.

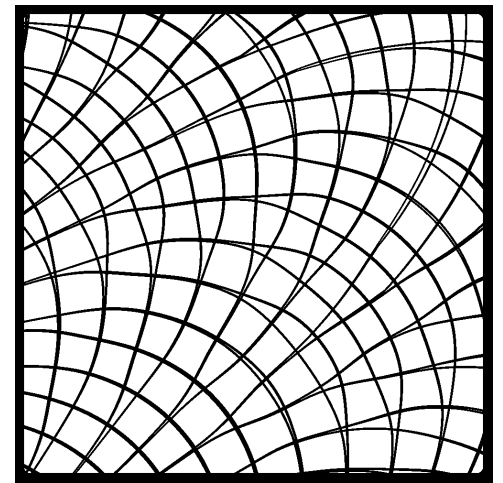

(c) $R^{*}=1.6 \varepsilon$

Figure 15: Projection on a fine mesh $\mathcal{T}^{f}$ using various radii for the convolution kernel $R^{*}$, using $\varepsilon=80 h^{f}$.

Finally, it has to be mentioned that using the adaptive periodicity projection approach the local unit-cell spacing is restricted to the interval $\left[\varepsilon^{-1 / 2}, \varepsilon^{1 / 2}\right]$, except in the transition zone where this can be in the range $\left[\varepsilon^{-3 / 2}, \varepsilon^{1 / 2}\right]$. The corresponding feature size of a member is thus bounded by $\left[\left(1-a_{u}\right) \varepsilon^{-3 / 2},\left(1-a_{l}\right) \varepsilon^{1 / 2}\right]$. Such a bound cannot be derived explicitly for the original mapping approach.

\section{Numerical examples for projection of coated structures}

The presented projection approach allows the coarse-scale optimized structure to be interpreted on a much finer mesh $\mathcal{T}^{f}$. We will demonstrate the performance of the projected designs $\mathcal{J}^{\phi}$, and compare these results to the compliance of the homogenization-based designs on the coarse mesh $\mathcal{J}^{c}$. Three different 
sources are identified that can lead to a difference between $\mathcal{J}^{\phi}$ and $\mathcal{J}^{c}$ : the effect of $h$-refinement, the interpolation of the coating from $\mathcal{T}^{c}$ onto $\mathcal{T}^{f}$, and the projection procedure. First, the effect of the former two will be discussed. Afterwards, various numerical examples will be used to demonstrate the potential of the projection approach in terms of both performance and computational cost.

\subsection{Effect of mesh refinement and interpolation of coating}

To test the effect of $h$-refinement, we interpolate the designs optimized with problem form 1 from Table 2 on a fine mesh $(3000 \times 1000$ elements $)$ using nearest-neighbor interpolation. The compliance values of the homogenization-based design on the coarse mesh $\mathcal{J}^{c}$ will be compared to the compliance values of the homogenization-based design on the fine mesh $\mathcal{J}^{f}$. Corresponding results are shown in Table 4.

Table 4: Compliance on coarse $\mathcal{J}^{c}$, and on fine mesh $\mathcal{J}^{f}$, for the MBB-beam example using the same settings as in Table 2, optimized with problem form 1 , for several infill densities $m^{I}$. Different interpolation methods for the coating are used.

\begin{tabular}{cccccccc}
\hline & Interpolation & $m^{I}=0.4$ & $m^{I}=0.5$ & $m^{I}=0.6$ & $m^{I}=0.7$ & $m^{I}=0.8$ & $m^{I}=0.9$ \\
\hline $\mathcal{J}^{c}$ & - & 318.45 & 291.14 & 274.92 & 266.85 & 251.38 & 236.37 \\
$\mathcal{J}^{f}$ & nearest-neighbor & 320.17 & 295.52 & 279.40 & 270.27 & 256.75 & 239.82 \\
$\mathcal{J}^{f}$ & linear & 328.34 & 305.32 & 286.30 & 273.18 & 258.39 & 239.09
\end{tabular}

As expected $\mathcal{J}^{f}$ is larger than $\mathcal{J}^{c}$ due to more accurate displacement calculation. Furthermore, the effect of the nearest-neighbor interpolation can be seen in Figure 16(a), where the jagged edges of the coating correspond to 10 elements. To avoid these jagged-edges we use a linear interpolation to map the normalized gradient norm $\|\nabla \tilde{\varphi}\|_{\alpha}$ onto the fine mesh. The corresponding values of $\mathcal{J}^{f}$ demonstrating the effect of this interpolation are shown in Table 2 as well.

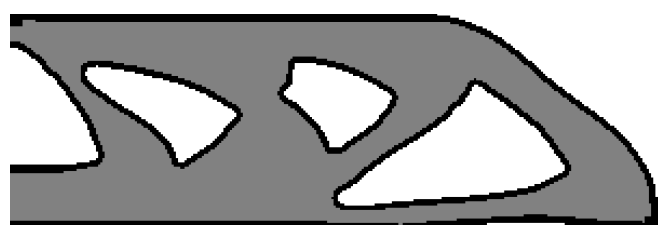

(a) nearest-neighbor, $\mathcal{J}^{f}=295.52$.

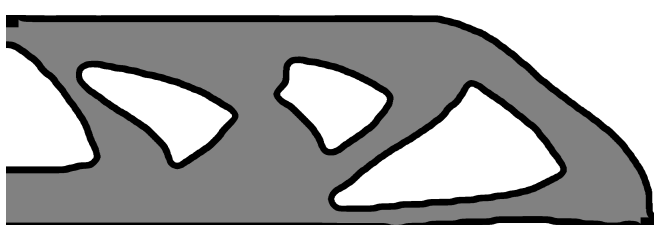

(b) linear, $\mathcal{J}^{f}=305.32$.

Figure 16: Density distribution for the MBB-beam example interpolated on a fine mesh of $3000 \times 1000$ elements, using different interpolation methods for $\|\nabla \tilde{\varphi}\|_{\alpha}$. The structure optimized using problem form 1 , with $m^{I}=0.5$.

It can be observed that interpolation of $\|\nabla \tilde{\varphi}\|_{\alpha}$ using nearest-neighbor interpolation leads to a better performance than linear interpolation. This seems rather counter-intuitive, since the latter method results in a smooth coating without jagged edges as can be seen in 16(b). However, it can be seen that the use of linear interpolation causes somewhat strange jumps close to the bottom right boundary condition, which is assumed to be the cause of the increased error between $\mathcal{J}^{c}$ and $\mathcal{J}^{f}$.

Despite a slightly increased compliance, linear interpolation is used for the mapping of $\|\nabla \tilde{\varphi}\|_{\alpha}$ onto $\mathcal{T}^{f}$. The reason is that the reference coating thickness is more uniform using this interpolation method. A future remedy can be to define the coating and interface on a much finer mesh than the coarse analysis mesh using a multi-resolution approach, e.g. (Nguyen et al., 2010; Groen et al., 2017).

\subsection{Effect of the different projection procedures}

To demonstrate the performance of the projection method, we consider the MBB-beam example optimized using problem form 1 , for $m^{I}=0.5$. The structure is projected on a fine mesh $(3000 \times 1000$ elements $)$, without the adaptive periodicity approach. We perform the projection for two different values of $\gamma, \gamma=10$ and $\gamma=10^{3}$ to demonstrate the effect of the angle constraint enforcement. Furthermore, we project the structure for 3 different average unit-cell sizes $\varepsilon$. The corresponding projected structures, the compliance $\mathcal{J}^{\phi}$ and the volume of the projected structures $V_{\phi}$ are shown in Figure 17. 


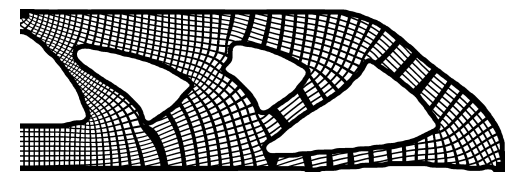

(a) $\varepsilon=20 h^{f}, \gamma=10, \mathcal{J}^{\phi}=315.56$ and $V_{\phi}=0.399$.

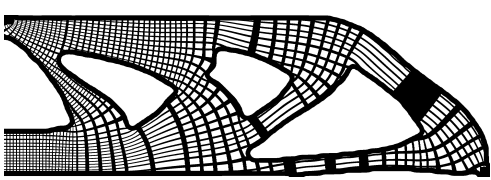

(d) $\varepsilon=20 h^{f}, \gamma=10^{3}, \mathcal{J}^{\phi}=307.93$ and $V_{\phi}=0.397$.

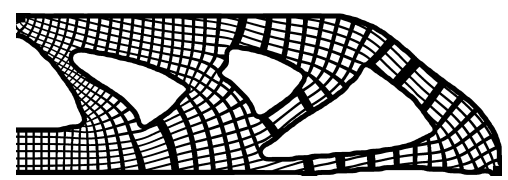

(b) $\varepsilon=30 h^{f}, \gamma=10, \mathcal{J}^{\phi}=316.48$ and $V_{\phi}=0.397$.

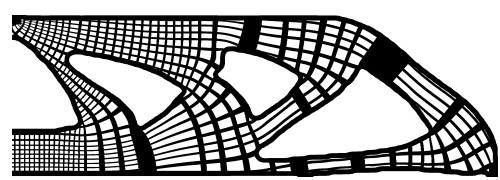

(e) $\varepsilon=30 h^{f}, \gamma=10^{3}, \mathcal{J}^{\phi}=304.79$ and $V_{\phi}=0.400$.

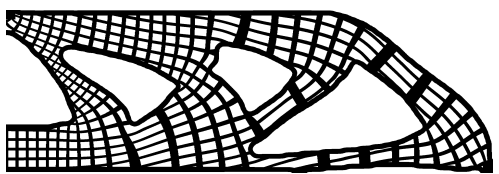

(c) $\varepsilon=40 h^{f}, \gamma=10, \mathcal{J}^{\phi}=319.62$ and $V_{\phi}=0.396$

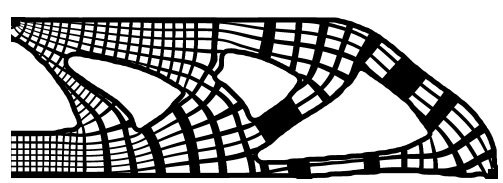

(f) $\varepsilon=40 h^{f}, \gamma=10^{3}, \mathcal{J}^{\phi}=301.99$ and $V_{\phi}=0.411$.

Figure 17: Projection of the MBB-beam example for a problem of type 1 , using $m^{I}=0.5$, on a fine mesh of $3000 \times 1000$ elements, where $\mathcal{J}^{f}=305.32$. No adaptive periodicity is used, results are shown for various values of angle constraint importance $\gamma$ and

From the bottom row it can be observed that a strong constraint enforcement $\left(\gamma=10^{3}\right)$ leads to structures performing very close to $\mathcal{J}^{f}$, i.e. within $1 \%$ ! Unfortunately however, we can identify a locally very distorted periodicity. These stretched unit-cells can lead to $V_{\phi}$ exceeding imposed volume constraint $V_{\max }$, and do not ensure a uniform infill. Lowering the angle constraint enforcement to $\gamma=10$, as is shown in the top row, yields much more regular infill patterns; however, at the cost of a slightly reduced performance.

A distorted periodicity can also lead to long thin members as can be seen in Figure 17(d). As was shown by Clausen et al. (2016) coated structures have an increased critical buckling load; however, thin unsupported infill members will reduce this desired effect by being prone to local buckling. Hence, regular projected unit-cells are required to retain a regular infill.

To use the best of both worlds, (exact angle enforcement, and regular unit-cells), we apply the adaptive periodicity projection approach as proposed in the previous section. The projected structures for $\gamma=10^{3}$ and different spacings can be seen in Figures 18(a)-(c).

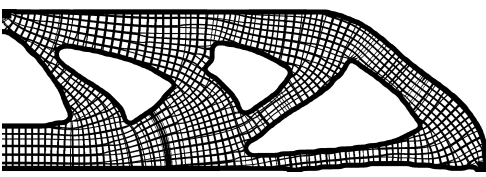

(a) $\varepsilon=20 h^{f}, \gamma=10^{3}, \mathcal{J}^{\phi}=307.58$ and $V_{\phi}=0.398$

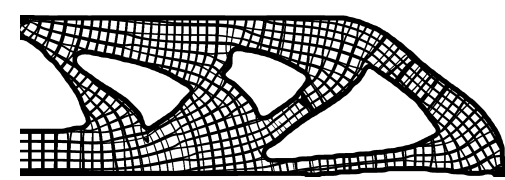

(d) $\varepsilon=30 h^{f}, \gamma=10^{2}, \mathcal{J}^{\phi}=304.58$ and $V_{\phi}=0.400$.

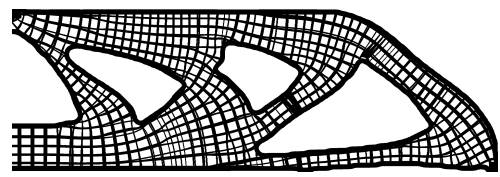

(b) $\varepsilon=30 h^{f}, \gamma=10^{3}, \mathcal{J}^{\phi}=306.41$ and $V_{\phi}=0.400$.

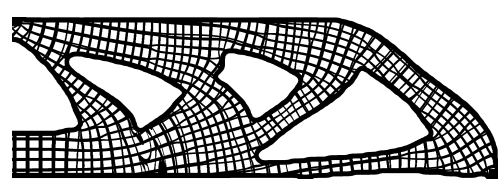

(e) $\varepsilon=30 h^{f}, \gamma=10^{1.5}, \mathcal{J}^{\phi}=308.67$ and $V_{\phi}=0.399$.

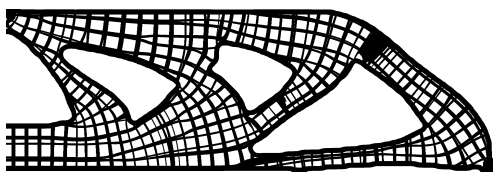

(c) $\varepsilon=40 h^{f}, \gamma=10^{3}, \mathcal{J}^{\phi}=301.23$ and $V_{\phi}=0.406$

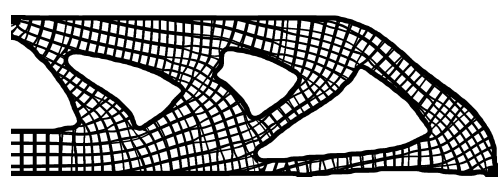

(f) $\varepsilon=30 h^{f}, \gamma=10, \mathcal{J}^{\phi}=320.22$ and $V_{\phi}=0.399$

Figure 18: Projection of the MBB-beam example for a problem of type 1 , using $m^{I}=0.5$, on a fine mesh of $3000 \times 1000$ elements, where $\mathcal{J}^{f}=305.32$. Adaptive periodicity projection is used, results are shown for various values of angle constraint importance $\gamma$ and $\varepsilon$.

It can be seen that values for $\mathcal{J}^{\phi}$ are again within $1 \%$ of $\mathcal{J}^{f}$, while a more uniform infill is maintained. Unfortunately, the proposed adaptive periodicity mapping procedure does not work perfectly yet when $\tilde{\lambda_{i}}$ is rapidly changing. This can be best seen in the top right of the MBB-beam shown in Figure 18(c), where the 
periodicity is locally undergoing a large change, such that there is no space to form nice and clear branches between different periodicities.

To prevent large jumps in periodicity the angle enforcement can be relaxed; therefore, the projection using $\varepsilon=30 h^{f}$ is shown for various values of $\gamma$ in Figures 18(d)-(f). Here it can be seen that $\gamma=10$, is the only value that completely prevents any of these local effects. Despite being slightly misaligned with the optimal orientation, the effect on the performance is small and the projected structures still perform within $5 \%$ of the homogenization-based designs.

To conclude, the proposed adaptive periodicity projection approach in combination with using $\gamma=10^{3}$ shows a clear potential. The structures perform almost identically to the homogenization-based designs; while the projected structures consist of near-regular infill. In future work, different formulations for adapting the periodicity in regions with large changes in periodicity will be further investigated, to improve this new and promising method even more.

\subsection{Comparison with coated structures optimized using SIMP}

To further demonstrate the performance of the approach proposed in this work, we compare it to the work of Wu et al. (2017). In this approach a coated structure is created, where the infill is optimized using a density-based approach as can be seen for the MBB-beam example in Figure 19 (a). Here, a discretization of $600 \times 200$ elements is used, with $R_{1}=0.15 L$ and $t_{r e f}=0.03 L$; furthermore, $V_{\max }=0.4, m^{I}=0.5$.

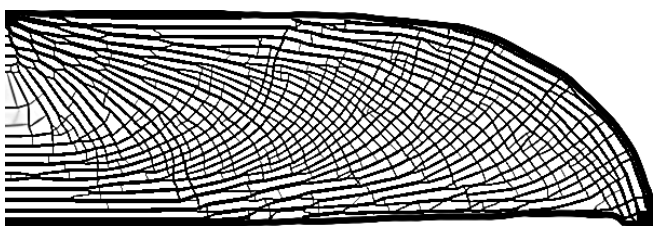

(a) Infill design using SIMP.

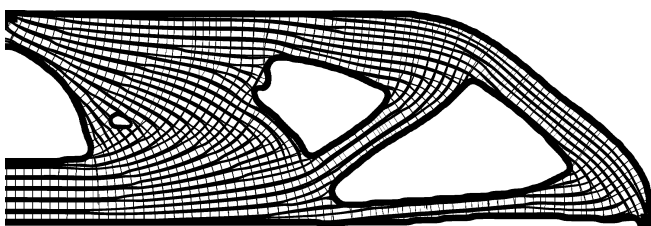

(b) Proposed projection procedure.

Figure 19: Comparison of the MBB-beam example using a SIMP-based approach (Wu et al., 2017), and the proposed projection based approach for the infill. Uniform infill density with $m^{I}=0.5$ is used while the microstructure is allowed to vary (problem form 2).

The method produces coated structures, where the infill has a locally uniform density, with no restriction on the freedom of the microstructure (close to an optimization problem of form 2 ). The optimization required a total time $T_{t o t}=292$ minutes using 600 design iterations. Hence, a more detailed design on a finer mesh is deemed computationally too expensive. The proposed projection procedure does not have a strong restriction on the level of detail of the projected shape, since the topology optimization can be performed on a relatively coarse mesh. The design optimized for problem form 2 , with $m^{I}=0.5$ and $a_{u}=0.9$ is projected on a fine mesh of $3000 \times 1000$ elements, using $\gamma=10^{3}, \varepsilon=20 h^{f}$ and the adaptive periodicity projection approach as can be seen in Figure 19(b).

For a fair comparison the structure optimized using the approach of Wu et al. (2017) is mapped on a fine mesh of $3000 \times 1000$ elements using nearest-neighbor interpolation. The compliance values for both approaches, for various infill densities $m^{I}$ are shown in Table 5 and Table 6 . Furthermore, a breakdown of the computational cost in optimization time $T_{o p t}$, mapping time $T_{\phi}$ as well as the total time $T_{t o t}$ is shown. It has to be noted that all simulations are done using a single processor MATLAB code on a standard PC running Windows 7.

It can be seen that the compliance values of the structures optimized using the mapping approach are in general lower than the compliance values obtained using the approach of $\mathrm{Wu}$ et al. (2017). The larger $\mathrm{m}^{I}$, the smaller the difference between the compliances obtained using both methods. Furthermore, it can be observed that a larger value of $\varepsilon$ results in a slightly larger volume of the projected structures, which results in a lower compliance. Another benefit of the projection approach is the clear solid and void structures, in contrast to elements with intermediate density from the infill optimized with SIMP (Wu et al., 2017). 
Table 5: Compliance values for optimization mesh $\mathcal{J}^{c}$ and for fine mesh $\mathcal{J}^{f}$, as well as volume fraction $V$ and optimization time $T_{\text {opt }}$ (shown in [hh:mm:ss]), for the MBB-beam example using the approach by Wu et al. (2017).

\begin{tabular}{ccccc}
\hline$m^{I}$ & $\mathcal{J}^{c}$ & $\mathcal{J}^{f}$ & $V$ & $T_{\text {tot }}$ \\
\hline 0.5 & 270.21 & 281.95 & 0.394 & $04: 52: 00$ \\
0.6 & 241.36 & 247.54 & 0.400 & $04: 58: 00$ \\
0.7 & 227.84 & 231.94 & 0.400 & $05: 00: 00$
\end{tabular}

Table 6: Compliance values for optimization mesh $\mathcal{J}^{c}$, fine mesh $\mathcal{J}^{f}$ and projected design $\mathcal{J}^{\phi}$, as well as volume fraction $V_{\phi}$ and time breakdown (shown in [hh:mm:ss]), for the MBB-beam example optimized using problem form 2 and projected using adaptive periodicity.

\begin{tabular}{ccccccccc}
\hline$m^{I}$ & $\varepsilon$ & $\mathcal{J}^{c}$ & $\mathcal{J}^{f}$ & $\mathcal{J}^{\phi}$ & $V_{\phi}$ & $T_{\text {opt }}$ & $T_{\phi}$ & $T_{\text {tot }}$ \\
\hline 0.5 & $20 h^{f}$ & 247.52 & 256.31 & 261.96 & 0.397 & $00: 23: 00$ & $00: 00: 20$ & $00: 23: 20$ \\
0.5 & $30 h^{f}$ & 247.52 & 256.31 & 260.68 & 0.401 & $00: 23: 00$ & $00: 00: 25$ & $00: 23: 25$ \\
0.5 & $40 h^{f}$ & 247.52 & 256.31 & 257.61 & 0.409 & $00: 23: 00$ & $00: 00: 30$ & $00: 23: 30$ \\
\hline 0.6 & $20 h^{f}$ & 234.30 & 242.53 & 242.03 & 0.400 & $00: 21: 19$ & $00: 00: 20$ & $00: 21: 39$ \\
0.6 & $30 h^{f}$ & 234.30 & 242.53 & 239.29 & 0.402 & $00: 21: 19$ & $00: 00: 25$ & $00: 21: 44$ \\
0.6 & $40 h^{f}$ & 234.30 & 242.53 & 239.21 & 0.404 & $00: 21: 19$ & $00: 00: 30$ & $00: 21: 49$ \\
\hline 0.7 & $20 h^{f}$ & 227.02 & 230.83 & 233.92 & 0.400 & $00: 21: 11$ & $00: 00: 19$ & $00: 21: 30$ \\
0.7 & $30 h^{f}$ & 227.02 & 230.83 & 231.26 & 0.404 & $00: 21: 11$ & $00: 00: 24$ & $00: 21: 35$ \\
0.7 & $40 h^{f}$ & 227.02 & 230.83 & 230.26 & 0.405 & $00: 21: 11$ & $00: 00: 29$ & $00: 21: 40$
\end{tabular}

However, the most important result is the computational efficiency of the proposed mapping method. Coated designs of high resolution (3 million elements!) can be obtained in less than half an hour, thanks to the proposed coarse scale homogenization-based optimization. While, the method by Wu et al. (2017) already requires close to 5 hours on a relatively coarse mesh of $(600 \times 200)$ elements. A reduction in computational cost of at least an order of magnitude can thus be obtained by the proposed approach, which allows for topology optimization as a more integrated part of the structural design process.

\subsection{Room for improvement}

As discussed in the previous section, the adaptive periodicity projection approach will restrict the local unit-cell spacing to the interval $\left[\varepsilon 2^{-1 / 2}, \varepsilon 2^{1 / 2}\right]$, except in the transition zone. When the angle changes slowly the microstructure is spaced in a very regular manner, as can be seen in the main load carrying member of Figure 20(a).

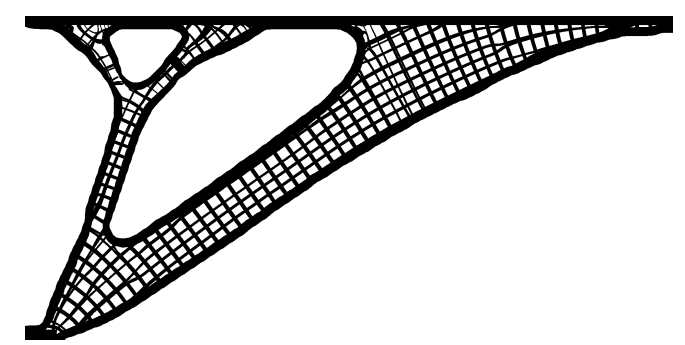

(a) Problem form 1, $\mathcal{J}^{f}=31.0874, \mathcal{J}^{\phi}=31.7504$ and $V_{\phi}=0.196$.

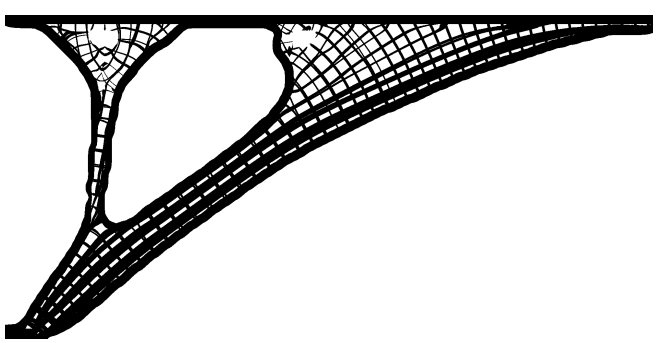

(b) Problem form $3, \mathcal{J}^{f}=26.4330, \mathcal{J}^{\phi}=26.8968$ and $V_{\phi}=0.198$.

Figure 20: Projected structures of the bridge example on a fine mesh of $2000 \times 1000$, elements using the adaptive periodicity approach, $\varepsilon=20 h^{f}$ and $\gamma=10^{3}$.

In the top left of the bridge-example the angle field is rapidly changing. Hence, there is no room for a smooth transition through all the periodicities and the spacing is slightly less regular as in the rest of the 
domain. A more worrying effect can be seen in the projected figure optimized for problem form 3, shown in 20(b). Here there is a singularity in the angle field below the void in the top left corner. Although the angle constraint ensures that most bars are well aligned with $\theta$, and the performance is within $2 \%$ of the homogenization-based performance, it can be seen that there is a jump in orientation of mapping functions $\phi_{i}$. We note that the observed singular points have low or negligible stresses, making the appearance less critical in terms of objective value. However, to avoid large changes in orientation and prevent the occurrence of singularities the angle field can possibly be regularized, e.g. using the approach presented by Allaire et al. (2018). We are confident that such a regularization will get $\mathcal{J}^{\phi}$ even closer to $\mathcal{J}^{f}$ and result in even more regular mapped designs.

\section{Concluding remarks}

An efficient approach to perform topology optimization of coated structures with orthotropic infill has been presented. Performing homogenization-based topology optimization allows for the modeling of designs with complex microstructures on a relatively coarse mesh, thus resulting in low computational cost. Furthermore, the double smoothing and projetion (DSP) approach ensures in almost all cases a clear distinction between coating, infill and void.

In the second part of the work, a projection approach is presented to map the coated designs from the assumption of infinite periodicity on a fine but realizable scale. A novel method to adaptively refine the periodicity is presented to allow for a regular spacing of the infill. Numerical experiments demonstrate that the projected designs, despite a lack of separation of scales, are very close (within 1-2\%) to the homogenizationbased performance. Furthermore, a comparison with (Wu et al., 2017) where the infill is optimized using a density-based method, shows that the projection procedure yields similar or even better performing designs at a finer resolution and at a computational cost which is at least 10 times lower, and potentially more in a case of mutual refinement.

This overall promising approach allows for extension of the method to 3D or to more complex loading situations. The main challenge here will lie in finding a parameterization that allows for smoothly varying microstructures through the domain. We are confident that such a parameterization can and will be found.

\section{Acknowledgments}

The authors acknowledge the financial support from the Villum Foundation (InnoTop VILLUM investigator project) and DTU Mechanical Engineering. Furthermore, the authors would like to express their gratitude to Anders Clausen for sharing his code on the optimization of coated structures. Finally, the authors wish to thank Krister Svanberg for the Matlab MMA code.

\section{References}

Allaire, G., Dapogny, C., Estevez, R., Faure, A., Michailidis, G., 2017. Structural optimization under overhang constraints imposed by additive manufacturing technologies. Journal of Computational Physics 351, 295 - 328. doi:10.1016/j.jcp. 2017.09 .041$.

Allaire, G., Geoffroy-Donders, P., Pantz, O., 2018. Topology optimization of modulated and oriented periodic microstructures by the homogenization method. Computers \& Mathematics with Applications doi:10.1016/j.camwa.2018.08.007.

Allaire, G., Jakabčin, L., 2018. Taking into account thermal residual stresses in topology optimization of structures built by additive manufacturing. Mathematical Models and Methods in Applied Sciences 28, 2313-2366. doi:10.1142/S0218202518500501.

Bendsøe, M., Kikuchi, N., 1988. Generating optimal topologies in structural design using a homogenization method. Computer Methods in Applied Mechanics and Engineering 71, 197-224. doi:10.1016/0045-7825(88)90086-2.

Christiansen, R.E., Lazarov, B.S., Jensen, J.S., Sigmund, O., 2015. Creating geometrically robust designs for highly sensitive problems using topology optimization. Structural and Multidisciplinary Optimization 52, 737-754. doi:10.1007/ s00158-015-1265-5.

Clausen, A., Aage, N., Sigmund, O., 2015. Topology optimization of coated structures and material interface problems. Computer Methods in Applied Mechanics and Engineering 290, 524 - 541. doi:10.1016/j.cma.2015.02.011.

Clausen, A., Aage, N., Sigmund, O., 2016. Exploiting additive manufacturing infill in topology optimization for improved buckling load. Engineering 2, 250 - 257. doi:10.1016/J.ENG.2016.02.006. 
Clausen, A., Andreassen, E., 2017. On filter boundary conditions in topology optimization. Structural and Multidisciplinary Optimization 56, 1147-1155. doi:10.1007/s00158-017-1709-1.

Clausen, A., Andreassen, E., Sigmund, O., 2017. Topology optimization of 3d shell structures with porous infill. Acta Mechanica Sinica 33, 778-791. doi:10.1007/s10409-017-0679-2.

Dapogny, C., Estevez, R., Faure, A., Michailidis, G., 2019. Shape and topology optimization considering anisotropic features induced by additive manufacturing processes. Computer Methods in Applied Mechanics and Engineering 344 , 626 - 665. doi:10.1016/j.cma.2018.09.036.

Gaynor, A.T., Guest, J.K., 2016. Topology optimization considering overhang constraints: Eliminating sacrificial support material in additive manufacturing through design. Structural and Multidisciplinary Optimization 54, 1157-1172. doi:10. $1007 / \mathrm{s} 00158-016-1551-\mathrm{x}$.

Gibson, L.J., Ashby, M.F., 1999. Cellular solids: structure and properties. Cambridge university press.

Groen, J.P., Langelaar, M., Sigmund, O., Ruess, M., 2017. Higher-order multi-resolution topology optimization using the finite cell method. International Journal for Numerical Methods in Engineering 110, 903-920. doi:10.1002/nme.5432.

Groen, J.P., Sigmund, O., 2017. Homogenization-based topology optimization for high-resolution manufacturable microstructures. International Journal of Numerical Methods in Engineering 113, 1148-1163. doi:10.1002/nme.5575.

Guest, J., Prévost, J., Belytschko, T., 2004. Achieving minimum length scale in topology optimization using nodal design variables and projection functions. International Journal for Numerical Methods in Engineering 61, 238-254. doi:10.1002/ nme.1064.

Hashin, Z., Shtrikman, S., 1963. A variational approach to the theory of the elastic behaviour of multiphase materials. Journal of the Mechanics and Physics of Solids 11, 127 - 140. doi:10.1016/0022-5096(63)90060-7.

Langelaar, M., 2017. An additive manufacturing filter for topology optimization of print-ready designs. Structural and Multidisciplinary Optimization 55, 871-883. doi:10.1007/s00158-016-1522-2.

Larsen, S.D., Sigmund, O., Groen, J.P., 2018. Optimal truss and frame design from projected homogenization-based topology optimization. Structural and Multidisciplinary Optimization 57, 1461-1474. doi:10.1007/s00158-018-1948-9.

Lazarov, B., Wang, F., Sigmund, O., 2016. Length scale and manufacturability in density-based topology optimization. Archive of Applied Mechanics 86, 189-218. doi:10.1007/s00419-015-1106-4.

Lazarov, B.S., Sigmund, O., 2011. Filters in topology optimization based on Helmholtz-type differential equations. International Journal for Numerical Methods in Engineering 86, 765-781. doi:10.1002/nme.3072.

Liu, J., Gaynor, A.T., Chen, S., Kang, Z., Suresh, K., Takezawa, A., Li, L., Kato, J., Tang, J., Wang, C.C.L., Cheng, L., Liang, X., To, A.C., 2018. Current and future trends in topology optimization for additive manufacturing. Structural and Multidisciplinary Optimization 57, 2457-2483. doi:10.1007/s00158-018-1994-3.

Lu, L., Sharf, A., Zhao, H., Wei, Y., Fan, Q., Chen, X., Savoye, Y., Tu, C., Cohen-Or, D., Chen, B., 2014. Build-to-last: Strength to weight 3d printed objects. ACM Trans. Graph. 33, 97:1-97:10. doi:10.1145/2601097.2601168.

Martínez, J., Dumas, J., Lefebvre, S., 2016. Procedural Voronoi foams for additive manufacturing. ACM Trans. Graph. 35, 44:1-44:12. doi:10.1145/2897824.2925922.

Nguyen, T., Paulino, G., Song, J., Le, C., 2010. A computational paradigm for multiresolution topology optimization (mtop). Structural and Multidisciplinary Optimization 41, 525-539. doi:10.1007/s00158-009-0443-8.

Pantz, O., Trabelsi, K., 2008. A post-treatment of the homogenization method for shape optimization. SIAM Journal on Control and Optimization 47, 1380-1398. doi:10.1137/070688900.

Pantz, O., Trabelsi, K., 2010. Construction of minimization sequences for shape optimization, in: 15th International Conference on Methods and Models in Automation and Robotics (MMAR), pp. 278-283. doi:10.1109/MMAR.2010.5587222.

Pedersen, P., 1989. On optimal orientation of orthotropic materials. Structural optimization 1, 101-106. doi:10.1007/ BF01637666.

Pedersen, P., 1990. Bounds on elastic energy in solids of orthotropic materials. Structural optimization 2, 55-63. doi:10.1007/ BF01743521.

Qian, X., 2017. Undercut and overhang angle control in topology optimization: A density gradient based integral approach. International Journal for Numerical Methods in Engineering 111, 247-272. doi:10.1002/nme.5461.

Ranjan, R., Yang, Y., Ayas, C., Langelaar, M., Van Keulen, F., 2017. Controlling local overheating in topology optimization for additive manufacturing, in: Proceedings of euspen special interest group meeting: additive manufacturing, Leuven, Belgium Google Scholar.

Sigmund, O., 2007. Morphology-based black and white filters for topology optimization. Structural and Multidisciplinary Optimization 33, 401-424. doi:10.1007/s00158-006-0087-x.

Sigmund, O., Aage, N., Andreassen, E., 2016. On the (non-)optimality of michell structures. Structural and Multidisciplinary Optimization 54, 361-373. doi:10.1007/s00158-016-1420-7.

Svanberg, K., 1987. The method of moving asymptotes-a new method for structural optimization. International Journal for Numerical Methods in Engineering 24, 359-373. doi:10.1002/nme.1620240207.

Vermaak, N., Michailidis, G., Parry, G., Estevez, R., Allaire, G., Bréchet, Y., 2014. Material interface effects on the topology optimization of multi-phase structures using a level set method. Structural and Multidisciplinary Optimization 50, 623-644. doi:10.1007/s00158-014-1074-2.

Wang, F., Lazarov, B., Sigmund, O., 2011. On projection methods, convergence and robust formulations in topology optimization. Structural and Multidisciplinary Optimization 43, 767-784. doi:10.1007/s00158-010-0602-y.

Wang, Y., Kang, Z., 2018. A level set method for shape and topology optimization of coated structures. Computer Methods in Applied Mechanics and Engineering 329, 553 - 574. doi:10.1016/j.cma.2017.09.017.

Wu, J., Aage, N., Westermann, R., Sigmund, O., 2018. Infill optimization for additive manufacturing - approaching bone-like porous structures. IEEE Transactions on Visualization and Computer Graphics 24, 1127-1140. doi:10.1109/TVCG.2017. 
2655523.

Wu, J., Clausen, A., Sigmund, O., 2017. Minimum compliance topology optimization of shell-infill composites for additive manufacturing. Computer Methods in Applied Mechanics and Engineering 326, 358 - 375. doi:10.1016/j.cma.2017.08.018.

Wu, J., Wang, C.C., Zhang, X., Westermann, R., 2016. Self-supporting rhombic infill structures for additive manufacturing. Computer-Aided Design 80, $32-42$. doi:10.1016/j.cad.2016.07.006.

Zhou, M., Lazarov, B.S., Wang, F., Sigmund, O., 2015. Minimum length scale in topology optimization by geometric constraints. Computer Methods in Applied Mechanics and Engineering 293, 266 - 282. doi:10.1016/j.cma.2015.05.003. 